\title{
MSSM Higgs boson phenomenology at the Fermilab Tevatron collider
}

\author{
M. Carena* \\ Fermi National Accelerator Laboratory, Batavia, Illinois 60510 \\ S. Mrenna ${ }^{\dagger}$ \\ Argonne National Laboratory, Argonne, Illinois 60439 \\ C. E. M. Wagner \\ CERN, TH Division, CH-1211 Geneva 23, Switzerland
}

(Received 4 September 1998; revised manuscript received 13 May 1999; published 10 September 1999)

\begin{abstract}
The Higgs sector of the minimal supersymmetric standard model (MSSM) consists of five physical Higgs bosons, which offer a variety of channels for their experimental search. In this study, we clarify the sensitivity of the future Fermilab Tevatron searches for neutral MSSM Higgs bosons in several channels as a function of the pseudoscalar mass $M_{A}$, $\tan \beta$, and the properties of the top and bottom squarks. We place special emphasis on the radiative corrections to the down-type quark and lepton couplings to the Higgs bosons for large $\tan \beta$, and how they effect production cross sections and branching ratios. [S0556-2821(99)00917-0]

PACS number(s): 14.80.Cp, 12.60.Jv, 14.80.Bn, 14.80.Ly
\end{abstract}

\section{INTRODUCTION}

The precision electroweak measurements performed at the CERN $e^{+} e^{-}$collider LEP, SLAC Large Detector (SLD) and the Fermilab Tevatron are consistent with the predictions of the standard model containing a light Higgs boson, with a mass of the order of the $Z$ boson mass. The searches for such a Higgs particle continue at the LEP and the Tevatron colliders. The searches at LEP2 $(\sqrt{s} \lesssim 200 \mathrm{GeV})$ are constrained by the collider energy, and a Higgs boson with standard model-like properties can be found only if its mass is below $105 \mathrm{GeV}[1]$.

The potential for discovering a light Higgs boson at the Tevatron collider when it is produced in association with a $W$ or $Z$ gauge boson has been discussed in several studies [2-6]. Although the kinematic reach of the Tevatron collider is much greater than for LEP2, the backgrounds to Higgs boson searches at hadron colliders are much larger than in $e^{+} e^{-}$machines. For this reason, a large integrated luminosity is essential to establish a signal at the Tevatron. Within the standard model, the general conclusion is that run II, with a total integrated luminosity of about $2 \mathrm{fb}^{-1}$ per detector, will be unable to extend the Higgs boson mass reach of LEP2. The main questions are what is the theoretical motivation for a Higgs boson with a mass slightly above the LEP2 reach, and what is the necessary upgrade in luminosity to cover that region?

We address the theoretical motivation by appealing to the minimal supersymmetric extension of the standard model (MSSM). The MSSM has the remarkable property that, for a sufficiently heavy supersymmetric spectrum, it fits to the precision electroweak observables as well as the standard model

\footnotetext{
*Email address: carena@fnal.gov

${ }^{\dagger}$ Email address: mrenna@hep.anl.gov

\#Email address: Carlos.Wagner@cern.ch
}

[7]. Moreover, the lightest $C P$-even Higgs boson mass $m_{h}$ is constrained to satisfy $m_{h} \lesssim 130 \mathrm{GeV}[8,9]$. The Higgs sector of this model consists of two Higgs doublets, with two $C P$-even Higgs bosons $h$ and $H$, one $C P$-odd Higgs boson $A$, and one charged Higgs boson $H^{ \pm}$. This richer spectrum allows for different production and decay processes at LEP and the Tevatron colliders than in the standard model.

In the supersymmetric limit, the neutral components of the two Higgs boson doublets $H_{1}$ and $H_{2}$ couple to downand up-type quarks, respectively. Lepton fields couple only to the $H_{1}$ Higgs boson. The MSSM, tree-level Yukawa couplings of the down quarks, leptons, and up quarks are related to their respective running masses by

$$
h_{d} \simeq \frac{m_{d}}{\mathrm{v} \cos \beta}, \quad h_{l} \simeq \frac{m_{l}}{\mathrm{v} \cos \beta}, \quad h_{u} \simeq \frac{m_{u}}{\mathrm{v} \sin \beta},
$$

where $\tan \beta=\mathrm{v}_{2} / \mathrm{v}_{1}$ is the ratio of the vacuum expectation values of the two Higgs doublets, and $v=\sqrt{\mathrm{v}_{1}^{2}+\mathrm{v}_{2}^{2}}$ $=174 \mathrm{GeV}$. In the standard model, only the top quark Yukawa coupling $h_{t}$ is of order 1 at the weak scale. In the MSSM, instead, the bottom and $\tau$ Yukawa couplings $h_{b}$ and $h_{\tau}$ can become of the same order as $h_{t}$, if $\tan \beta$ is sufficiently large. This can have important phenomenological consequences.

Quite generally, the two $C P$-even Higgs boson eigenstates are a mixture of the real, neutral $H_{1}$ and $H_{2}$ components

$$
\left(\begin{array}{l}
h \\
H
\end{array}\right)=\left(\begin{array}{cc}
-\sin \alpha & \cos \alpha \\
\cos \alpha & \sin \alpha
\end{array}\right)\left(\begin{array}{c}
H_{1}^{0} \\
H_{2}^{0}
\end{array}\right)
$$

and the lightest $C P$-even Higgs boson couples to down quarks (leptons), and up quarks by its standard model values times $-\sin \alpha / \cos \beta$ and $\cos \alpha / \sin \beta$, respectively. The cou- 
plings to the heavier $C P$-even Higgs boson are given by the standard model values times $\cos \alpha / \cos \beta$ and $\sin \alpha / \sin \beta$, respectively. Analogously, the coupling of the $C P$-odd Higgs boson to down quarks (leptons) and up quarks is given by the standard model coupling times $\tan \beta$ and $1 / \tan \beta$, respectively. Moreover, the lightest (heaviest) $C P$-even Higgs boson has $Z Z h$ and $W W h$ ( $Z Z H$ and $W W H$ ) couplings which are given by the standard model value times $\sin (\beta$ $-\alpha)[\cos (\beta-\alpha)]$, while it can be produced in association with a $C P$-odd Higgs boson with a ZhA (ZHA) coupling which is proportional to $\cos (\beta-\alpha)[\sin (\beta-\alpha)]$.

For sufficiently large values of the $C P$-odd Higgs boson mass $m_{A}$, the effective theory at low energies contains only one Higgs doublet, with standard model-like properties, in the combination ${ }^{1}$

$$
h \simeq \phi^{\mathrm{SM}}=H_{1}^{0} \cos \beta+H_{2}^{0} \sin \beta, \quad\left\langle\phi^{\mathrm{SM}}\right\rangle=\mathrm{v}
$$

where $\sin \alpha \simeq-\cos \beta, \cos \alpha \simeq \sin \beta$, and hence $\sin ^{2}(\beta-\alpha)$ $=1$. In this limit, if all supersymmetric particles are heavy, then all phenomenological conclusions drawn for a SM Higgs boson are robust when extended to the the lightest $C P$-even Higgs boson of the MSSM, which, as mentioned before, is at the same time constrained to have a mass below about $130 \mathrm{GeV}$. For large $\tan \beta$, one of the $C P$-even neutral Higgs bosons tends to be degenerate in mass with the $C P$-odd Higgs boson and couples strongly to the bottom quark and tau lepton. The other $C P$-even Higgs boson has standard model-like couplings to the gauge bosons, while its coupling to the down quarks and leptons may be highly nonstandard. If $m_{A}$ is large, then $h$ is the Higgs boson with SM-like properties as described above. If $m_{A}$ is small, then $H$ is the one with SM-like couplings to the gauge bosons. In the following, the symbol $\phi$ denotes a generic Higgs boson.

In this article, we analyze the discovery potential of the Tevatron collider for MSSM Higgs bosons in different production channels. Section II discusses the signals from Higgs boson production in association with weak gauge bosons and their dependence on the MSSM parameter space. Section III contains details of Yukawa coupling effects for large $\tan \beta$. Section IV deals with the phenomenological implications of these effects for signals in the $V \phi(V=W$ or $Z)$ and $b \bar{b} \phi$ production channels. In Sec. V, we consider the correlation between the bottom mass corrections and the supersymmetric contributions to the branching ratio $B(b \rightarrow s \gamma)$. Finally, Sec. VI is reserved for our conclusions.

\footnotetext{
${ }^{1}$ In the following, we shall use the term "standard model-like Higgs boson" $\phi^{\mathrm{SM}}$, more generically, to refer to a Higgs boson with standard model couplings to the gauge bosons, with no implication about its couplings to fermions. To avoid confusions, whenever we refer to simulations performed within the standard model, we shall denote the standard model Higgs boson as $H^{\mathrm{SM}}$.
}

\section{MSSM PHENOMENOLOGY OF THE $W \phi$ AND $Z \phi$ CHANNELS}

The production of $W \phi$, followed by the decays $W\left(\rightarrow e \nu_{e}, \mu \nu_{\mu}\right)$ and $\phi(\rightarrow b \bar{b})$, and the production of $Z \phi$, followed by the decays $Z(\rightarrow \nu \bar{\nu})$ or $Z\left(\rightarrow e^{+} e^{-}, \mu^{+} \mu^{-}\right)$and $\phi(\rightarrow b \bar{b})$, are the gold-plated search modes for the standard model Higgs boson $\phi=H^{\mathrm{SM}}$ at the Tevatron collider, while LEP2 is sensitive to the $Z \phi$ process. Additionally, the allhadronic decays of $W \phi+Z \phi$ may extend the reach, and the Higgs boson decay $\phi\left(\rightarrow \tau^{+} \tau^{-}\right)$or $\phi\left(\rightarrow W^{*} W^{*}\right)$ may be observable. However, there are several, unresolved experimental issues concerning these other channels that require detailed study by the experimental collaborations, and we do not consider them in this section.

To quantify the experimental reach in a model independent way, it is useful to consider the function

$$
R=\frac{\sigma(p \bar{p} \rightarrow V \phi)}{\sigma\left(p \bar{p} \rightarrow V H^{\mathrm{SM}}\right)} \frac{B(\phi \rightarrow b \bar{b})}{B\left(H^{\mathrm{SM}} \rightarrow b \bar{b}\right)},
$$

where $\sigma$ denotes a production cross section, $B$ denotes a branching ratio, $H^{\mathrm{SM}}$ represents a standard model Higgs boson, and $V=W$ or $Z$. In the MSSM, the ratio of cross sections is just given by $\sin ^{2}(\beta-\alpha)$ or $\cos ^{2}(\beta-\alpha)$ depending on $\phi$ being the lightest or heaviest $C P$-even Higgs boson, respectively, ${ }^{2}$ while the ratio of branching ratios has a more complicated behavior. Barring the possibility of large nextto-leading-order (NLO), SUSY corrections to the $V V \phi$ vertex, there is no enhancement of the production cross section in the MSSM over the standard model. On the other hand, the branching ratio to $b \bar{b}$ and $\tau^{+} \tau^{-}$final states are affected by the factors $-\sin \alpha / \cos \beta$ for $h$ and $\cos \alpha / \cos \beta$ for $H$ over the SM Higgs boson couplings. These factors can produce an increase or decrease of the MSSM coupling of the Higgs boson to bottom quarks, depending on the value of the $C P$-odd mass, $\tan \beta$, and the top and bottom squark mass parameters. Additional corrections can arise at large $\tan \beta$, which will be discussed in Secs. III and IV. In this study, the Higgs boson properties are calculated using the program HDECAY $[10]^{3}$

As mentioned above, for large $m_{A}$, the low-energy, effective theory contains only one Higgs boson with SM-like properties. The Yukawa couplings tend to the standard model values, and $\sin ^{2}(\beta-\alpha) \simeq 1$. As long as no new decay modes are open, $h$ has the same properties as $H^{\mathrm{SM}}$, and a discovery or exclusion limit for a standard model Higgs boson applies equally well to $h$, and $R=1$. If Higgs boson decays to sparticles become important, then it is quite likely that additional Higgs boson production modes exist and enhance the potential signal, rather than decrease it. For ex-

\footnotetext{
${ }^{2}$ Observe that $\sin (\beta-\alpha)[\cos (\beta-\alpha)]$ denotes the component of the lightest (heaviest) $C P$-even Higgs boson in the combination which acquires a vacuum expectation value $v$ in Eq. (1.3).

${ }^{3} \mathrm{We}$ have trivially modified the program to incorporate the large $\tan \beta$ corrections to be discussed in later sections.
} 
ample, if the sparticle spectrum is of the order of $m_{h}$, then processes such as $p \bar{p} \rightarrow N_{2}\left(\rightarrow N_{1} h\right) C_{1}\left(\rightarrow N_{1} W\right)+X$ can occur. In our analysis, we shall always consider the limit of heavy sparticle masses, where such supersymmetric contributions to the Higgs production and decay processes are negligible.

In the large $m_{A}$ limit, the renormalization-group improved result for the lightest Higgs boson mass, including two-loop leading-log effects $[8,9,11,12]$, has the approximate analytic form [8]

$$
\begin{gathered}
m_{h}^{2} \equiv m_{\phi^{S M}}^{2}=M_{Z}^{2} \cos ^{2}(2 \beta)\left(1-\frac{3}{8 \pi^{2}} \frac{m_{t}^{2}}{\mathrm{v}^{2}} t\right)+\frac{3}{4 \pi^{2}} \frac{m_{t}^{4}}{\mathrm{v}^{2}} \\
\times\left[\frac{1}{2} \tilde{X}_{t}+t+\frac{1}{16 \pi^{2}}\left(\frac{3}{2} \frac{m_{t}^{2}}{\mathrm{v}^{2}}-32 \pi \alpha_{3}\right)\left(\tilde{X}_{t} t+t^{2}\right)\right] \\
\tilde{X}_{t}=2 \tilde{a}^{2}\left(1-\frac{\tilde{a}^{2}}{12}\right), \quad \tilde{a}=\bar{A}_{t}-\bar{\mu} / \tan \beta,
\end{gathered}
$$

where $\bar{\mu}=\mu / M_{S}$ and $\bar{A}_{t}=A_{t} / M_{S}$, with $M_{S}^{2}=\left(m_{\tilde{t}_{2}}^{2}+m_{\tilde{t}_{1}}^{2}\right) / 2$, and $t=\log \left(M_{S}^{2} / m_{t}^{2}\right)$. The above formula is based on an approximation in which the right-handed and left-handed top squark supersymmetry breaking parameters are assumed to be close in value, and hence the top squark mass splitting is induced by the mixing parameter $m_{t} \tilde{a} \times M_{S}$. Moreover, this expression is based on an expansion in powers of $m_{t} \tilde{a} / M_{S}$ and is valid only if $\left(\left|m_{\tilde{t}_{1}}^{2}-m_{\tilde{t}_{2}}^{2}\right|\right) /\left(m_{\tilde{t}_{2}}^{2}+m_{\tilde{t}_{1}}^{2}\right)<0.5$, where $m_{\tilde{t}_{1}}$ and $m_{\tilde{t}_{2}}$ are the lightest and heaviest top squark mass eigenstates. This simplified expression is very useful for understanding the results of this work, although we go beyond this approximation [8] in our full analysis. Finally, in the above, we have ignored corrections induced by the sbottom sector, which, as we shall discuss below, may become relevant for very large values of $\tan \beta$.

The value of $m_{h}$ in Eq. (2.2) is maximized for large values of $\tan \beta$ and $M_{S}$ and $\tilde{a}^{2}=6$. Because of dependence of the lightest $C P$-even Higgs mass on $\tan \beta$, LEP will be able to probe the low $\tan \beta$ region of the MSSM. Indeed, recent analyses suggest that even the present, relatively low bounds on a standard model-like Higgs boson from LEP2 have strong implications for the minimal supergravity model [13]. Moreover, it has been shown that, for large $m_{A}$, LEP2 will probe values of $\tan \beta \lesssim 2$ for arbitrary values of the top squark masses and mixing angles [14]. Since, in general, the lower bound on $\tan \beta$ is expected to be obtained for large values of $m_{A}$, the absence of a Higgs signal in the $Z Z h$ channel at LEP2 will provide a strong motivation for models with moderate or large values of $\tan \beta$.

For lower values of the $C P$-odd Higgs boson mass, $\sin ^{2}(\beta-\alpha)$ can take any value between 0 and 1 , and it is a model-dependent question whether $h$ or $H$ produces a viable signal in the $V \phi$ production channel. For moderate or large values of $\tan \beta \geq 5$, it is easy to identify the main properties of the $C P$-even Higgs sector. More specifically, three cases may occur.

(a) If $m_{A}<m_{\phi} \mathrm{SM}$, then $\sin \alpha \simeq-1, \cos \alpha \simeq \mathcal{O}(1 / \tan \beta)$, and $\cos (\beta-\alpha) \simeq 1$. In this case, the heaviest $C P$-even Higgs boson has a production rate which is similar to the standard model case. The branching ratio of the decay into bottom quarks and $\tau$ leptons, however, can become highly nonstandard, since $\cos \alpha$ and $\cos \beta$, may differ by a factor of order 1 .

(b) If $m_{A}>m_{\phi} \mathrm{sm}$, then the lightest $C P$-even Higgs boson has a production rate similar to the standard model case. For $m_{A} \gg m_{\phi} \mathrm{SM}$, the branching ratio of the decay of this Higgs boson into down quarks is standard model-like. However, when $m_{A}$ becomes close to $m_{\phi} \mathrm{SM}$, there can be important differences in the branching ratios with respect to the SM ones.

(c) If $m_{A} \simeq m_{\phi} \mathrm{SM}$, then $\sin ^{2}(\beta-\alpha) \simeq \cos ^{2}(\beta-\alpha) \simeq \mathcal{O}(0.5)$, and the couplings of both neutral $C P$-even Higgs bosons to bottom quarks tend to be highly nonstandard.

To better understand the behavior of the Higgs boson branching ratios in these different cases, we analyze the Higgs boson mass matrix. Assuming the approximate conservation of $C P$ in the Higgs sector, the $C P$-even Higgs boson masses may be determined by diagonalizing the 2 $\times 2$ symmetric mass matrix $\mathcal{M}^{2}$. After including the dominant one-loop corrections induced by the top and bottom squark sectors, together with the two-loop, leading-logarithm effects, the elements of $\mathcal{M}^{2}$ are [8]

$$
\begin{aligned}
\mathcal{M}_{11}^{2} \simeq & m_{A}^{2} \sin ^{2} \beta+M_{Z}^{2} \cos ^{2} \beta-\frac{h_{t}^{4} \mathrm{v}^{2}}{16 \pi^{2}} \bar{\mu}^{2} \sin ^{2} \beta \tilde{a}^{2} \\
& \times\left[1+\frac{t}{16 \pi^{2}}\left(6 h_{t}^{2}-2 h_{b}^{2}-16 g_{3}^{2}\right)\right]+\mathcal{O}\left(h_{t}^{2} M_{Z}^{2}\right) \\
& -\frac{h_{b}^{4} \mathrm{v}^{2}}{16 \pi^{2}} \bar{\mu}^{2} \sin ^{2} \beta \bar{A}_{b}^{2}\left[1+\frac{t}{16 \pi^{2}}\left(6 h_{b}^{2}-2 h_{t}^{2}-16 g_{3}^{2}\right)\right], \\
\mathcal{M}_{22}^{2} \simeq & m_{A}^{2} \cos ^{2} \beta+M_{Z}^{2} \sin ^{2} \beta\left(1-\frac{3}{8 \pi^{2}} h_{t}^{2} t\right) \\
& +\frac{h_{t}^{4} \mathrm{v}^{2}}{16 \pi^{2}} 12 \sin ^{2} \beta\left\{t\left[1+\frac{t}{16 \pi^{2}}\left(1.5 h_{t}^{2}+0.5 h_{b}^{2}-8 g_{3}^{2}\right)\right]\right. \\
+ & \left.\bar{A}_{t} \tilde{a}\left(1-\frac{\bar{A}_{t} \tilde{a}}{12}\right)\left[1+\frac{t}{16 \pi^{2}}\left(3 h_{t}^{2}+h_{b}^{2}-16 g_{3}^{2}\right)\right]\right\} \\
& -\frac{\mathrm{v}^{2} h_{b}^{4}}{16 \pi^{2}} \sin ^{2} \beta \bar{\mu}^{4}\left[1+\frac{t}{16 \pi^{2}}\left(9 h_{b}^{2}-5 h_{t}^{2}-16 g_{3}^{2}\right)\right] \\
& \\
& \\
&
\end{aligned}
$$




$$
\begin{aligned}
\mathcal{M}_{12}^{2} \simeq & -\left[m_{A}^{2}+M_{Z}^{2}-\frac{h_{t}^{4} \mathrm{v}^{2}}{8 \pi^{2}}\left(3 \bar{\mu}^{2}-\bar{\mu}^{2} \bar{A}_{t}^{2}\right)\right] \sin \beta \cos \beta \\
& +\left[\frac{h_{t}^{4} \mathrm{v}^{2}}{16 \pi^{2}} \sin ^{2} \beta \bar{\mu} \tilde{a}\left[\bar{A}_{t} \tilde{a}-6\right]+\frac{3 h_{t}^{2} M_{Z}^{2}}{32 \pi^{2}} \bar{\mu} \tilde{a}\right] \\
& \times\left[1+\frac{t}{16 \pi^{2}}\left(4.5 h_{t}^{2}-0.5 h_{b}^{2}-16 g_{3}^{2}\right)\right] \\
& +\frac{h_{b}^{4} \mathrm{v}^{2}}{16 \pi^{2}} \sin ^{2} \beta \bar{\mu}^{3} \bar{A}_{b} \\
& \times\left[1+\frac{t}{16 \pi^{2}}\left(7.5 h_{b}^{2}-3.5 h_{t}^{2}-16 g_{3}^{2}\right)\right]
\end{aligned}
$$

where $g_{3}$ is the QCD running coupling constant and $\bar{A}_{b}$ $=A_{b} / M_{S}$. In the above, we have retained only the leading terms in powers of $h_{b}$ and $\tan \beta$. We have also included the small, $\mathcal{O}\left(h_{t}^{2} M_{Z}^{2}\right)$ correction to $\mathcal{M}_{12}^{2}$ explicitly because it plays a relevant role in our analysis. The above expressions hold only in the limit of small splittings between the running top squark masses. Moreover, the condition $2 m_{t} \max \left(\left|A_{t}\right|,|\mu|\right)<M_{S}^{2}$ must be fulfilled. Similar conditions should hold in the sbottom sector. The leading two-loop logarithmic corrections to the squared Higgs mass matrix elements included above can be as large as $10-20 \%$ when supersymmetric particles are heavy, and are very relevant in determining the Higgs boson mass eigenvalues and mixing angles. Observe that Eq. (2.2) may be easily obtained from the above expression, ${ }^{4}$ by computing the determinant of the Higgs boson mass matrix and setting the heavy $C P$-even Higgs boson mass approximately equal to $m_{A}$.

The mixing angle $\alpha$ can be determined from the expression

$$
\sin \alpha \cos \alpha=\frac{\mathcal{M}_{12}^{2}}{\sqrt{\left(\operatorname{Tr} \mathcal{M}^{2}\right)^{2}-4 \operatorname{det} \mathcal{M}^{2}}} .
$$

In the limit that $\mathcal{M}_{12}^{2} \rightarrow 0$, either $\sin \alpha$ or $\cos \alpha \rightarrow 0$. For moderate or large values of $\tan \beta$, if case (a) is realized and $\cos \alpha \simeq 0$, the coupling of the standard model-like Higgs boson $H$ to $b \bar{b}$ and $\tau^{+} \tau^{-}$is diminished, and decays to $g g, \gamma \gamma$, $c \bar{c}$, and $W^{(*)} W^{(*)}$ can be greatly enhanced over standard model expectations [15]. The same can happen for $h$ in case (b) when $\sin \alpha \simeq 0$. For moderate or large values of $\tan \beta$, the vanishing of $\mathcal{M}_{12}^{2}$ leads to the approximate numerical relation:

\footnotetext{
${ }^{4}$ There is a slight discrepancy in the subdominant, Yukawadependent, two-loop, leading-logarithmic corrections, which is due to the fact that the expressions written above are only strictly valid for values of the $C P$-odd mass of the order of the weak scale.
}

$$
\begin{aligned}
& {\left[\frac{m_{A}^{2}}{m_{Z}^{2}}-\frac{1}{2 \pi^{2}}\left(3 \bar{\mu}^{2}-\bar{\mu}^{2} \bar{A}_{t}^{2}\right)+1\right] } \\
\simeq & \frac{\tan \beta}{100}\left[\bar{\mu} \tilde{a}\left(2 \tilde{a} \bar{A}_{t}-11\right)\right]\left[1-\frac{15}{16 \pi^{2}} t\right],
\end{aligned}
$$

where we have assumed, for simplicity, that $\bar{A}_{b} \simeq 0$ (an assumption we shall always make in the following), ${ }^{5}$ we have also neglected all other bottom Yukawa coupling effects and replaced $h_{t}$ and $g_{3}$ and the weak gauge couplings by their approximate numerical values at the weak scale.

For low values of $m_{A}$, or large values of the mixing parameters, a cancellation can easily take place for large values of $\tan \beta$. For instance, if $M_{S} \simeq 1 \mathrm{TeV}, \bar{\mu}=-\tilde{a}=1$, and $m_{A}$ $\simeq 80 \mathrm{GeV}$, a cancellation can take place for $\tan \beta \simeq 28$, with the spectrum $m_{A} \simeq m_{h}$ and $m_{H} \simeq 117 \mathrm{GeV}$. The heaviest $C P$-even Higgs boson has standard model-like couplings to the gauge bosons $\left[\cos ^{2}(\beta-\alpha) \simeq 1\right]$, but the branching ratios for decays into $W^{ \pm}$bosons, gluons, and charm quarks are enhanced with respect to the SM case: $B_{W}=0.34, B_{g}=0.27$, and $B_{c}=0.11$. For the same value of $m_{A}$, but larger values of the stop mixing parameters, $\bar{\mu}=\tilde{a}=\sqrt{7}$ (at the edge of the region of validity of the above approximation), an approximate cancellation of the tree-level bottom and $\tau$ lepton couplings is achieved for $\tan \beta=20$, for which $m_{H} \simeq 124 \mathrm{GeV}$, with branching ratios $B_{W}=0.57, B_{g}=0.21$, and $B_{c}=0.08$.

An interesting point is that, for large values of $\tan \beta$ and values of the top squark mixing parameters which maximize $\mathcal{M}_{22}^{2}, \quad(\tilde{a}=\sqrt{6})$, the dominant, $m_{t}^{4}$-dependent corrections to the off-diagonal elements of the Higgs boson matrix vanish. In this case, the corrections to $\mathcal{M}_{12}^{2}$ are dominated by the $m_{t}^{2} M_{Z}^{2}$ dependent terms [see Eq. (2.3)], which, for $M_{S}$ $\simeq 1 \mathrm{TeV}$, cannot be large enough to induce an approximate cancellation of the off-diagonal terms for $|\bar{\mu}| \leqslant 1$ in the region of $m_{A} \lesssim 400 \mathrm{GeV}$ and $\tan \beta \lesssim 50$ considered in this article. However, even for $|\bar{\mu}|=1$, the impact of the radiative corrections to the off-diagonal elements of the Higgs boson mass matrix may be very relevant for low values of $m_{A}$, and we expect large variations of the branching ratio of the decay of the heaviest $C P$-even Higgs boson into bottom quarks with respect to the choice of the sign of $\mu$ in this region of parameters.

Moreover, away from $|\tilde{a}|=\sqrt{6}$, the $m_{t}^{4}$-dependent radiative corrections to $\mathcal{M}_{12}^{2}$ depend strongly on the sign of $\bar{\mu}$ $\times \bar{A}_{t}\left(\bar{A}_{t} \simeq \tilde{a}\right.$ for large $\tan \beta$ and moderate $\left.\mu\right)$ and on the

\footnotetext{
${ }^{5}$ Varying the value of $A_{b}$ has negligible impact on the value of $m_{\phi^{\mathrm{SM}}}\left(m_{\phi^{\mathrm{SM}}}^{2} \simeq \mathcal{M}_{22}^{2}\right)$, while it modifies the precise value of the Higgs mixing angles only for very large values of $\tan \beta$ and small values of $m_{A}$. We have explicitly checked that there are only small variations in our results, for values of $\tan \beta \geq 30$, when varying $\bar{A}_{b}$ from $\bar{A}_{b}=0$ to $\bar{A}_{b}= \pm 1$. Very large values of $\left|\bar{A}_{b}\right|, \bar{\mu}$ and $\tan \beta$ may, however, lead to relevant effects in the Higgs mixing angle, as follows from Eq. (2.3).
} 
value of $\left|\bar{A}_{t}\right|$. For the same value of $\tilde{a}$, a change in the sign of $\mu$ can lead to observable variations in the branching ratio for the Higgs boson decay into bottom quarks. If $\left|\bar{A}_{t}\right| \lesssim \sqrt{11 / 2}$, the absolute value of the off-diagonal matrix element, and hence, the coupling of bottom quarks to the standard modellike Higgs boson tends to be suppressed (enhanced) for values of $\bar{\mu} \times \bar{A}_{t}<0 \quad\left(\bar{\mu} \times \bar{A}_{t}>0\right)$. For larger values of $\left|\bar{A}_{t}\right|$, instead, the suppression (enhancement) occurs for the opposite sign of $\bar{\mu} \times \bar{A}_{t}$. Finally, it is important to stress that, in the large $\tan \beta$ regime, extra corrections to the Yukawa couplings may be important depending on the MSSM spectrum, and we shall come back to this topic later in Sec. III.

Numerical results for the $V h$ and $V H$ channels. The starting point for a numerical analysis of the MSSM Higgs boson phenomenology is an understanding of the standard model Higgs boson phenomenology. There have been several studies of the expected signals and backgrounds, and refinements to those studies [2-6,16-18]. For our numerical results, we rely on the detailed simulations performed by the Higgs Working Group of the Physics at Run II - Supersymmetry/ Higgs Workshop [19] for the $V H^{\mathrm{SM}}$ channel. The exact results assume a combination of CDF and D0 data, a $10 \% b \bar{b}$ mass resolution and systematic errors. ${ }^{6}$

Given the signal and background estimates for a standard model Higgs boson mass, we can determine which values of $R$ would lead to a discovery with a $5 \sigma$ significance $(\sigma$ $=S / \sqrt{B})$ or a $95 \%$ C.L. $(1.64 \sigma)$ exclusion. If the $R$-contour lies below $R=1$, then a standard model-like Higgs boson could be discovered or excluded. The exclusion potential of the $V \phi$ channel at the Tevatron is summarized in Fig. 1, while the analogous discovery potential is described in Fig. 2. Figure 1 shows the $95 \%$ C.L. exclusion limit as a function of $M_{\phi}$ for LEP2 running at $\sqrt{s}=198 \mathrm{GeV}$ and collecting $200 \mathrm{pb}^{-1}$ of data per experiment (hatched region), and for the Tevatron with $30 \mathrm{fb}^{-1}$ (solid), $10 \mathrm{fb}^{-1}$ (long-dash), and $2 \mathrm{fb}^{-1}$ (short-dash) per experiment. With $10 \mathrm{fb}^{-1}$, if no signal were found, the Tevatron could test a standard model Higgs boson $(R=1)$ with mass below about $125 \mathrm{GeV}$, whereas with $2 \mathrm{fb}^{-1}$ of data the exclusion limit will be comparable to that of LEP2. Figure 2 shows $5 \sigma$ discovery curves as a function of $M_{\phi}$ for LEP2 and the Tevatron under the same assumptions as for Fig. 1. With $30 \mathrm{fb}^{-1}$ (solid) of data a SM Higgs boson of mass below $125 \mathrm{GeV}$ can be discovered in this channel.

It is convenient to present MSSM Higgs boson results in the $m_{A}-\tan \beta$ plane. To perform the MSSM analysis, we specify the parameters of the top and bottom squarks and calculate the function $R$ at each value of $m_{A}$ and $\tan \beta$. If $R$ is larger than the value at the same Higgs boson mass in Fig. 1 or Fig. 2 (for a given integrated luminosity), then the point

\footnotetext{
${ }^{6}$ For these studies the systematic errors are taken into account, incorporating a relative uncertainty on the background for each channel, which is the smaller of $10 \%$ of the expected background or $1 / \sqrt{L B}$, where $L$ is the total integrated luminosity in units of $\mathrm{fb}^{-1}$ and $B$ is the expected number of background events in $1 \mathrm{fb}^{-1}$.
}

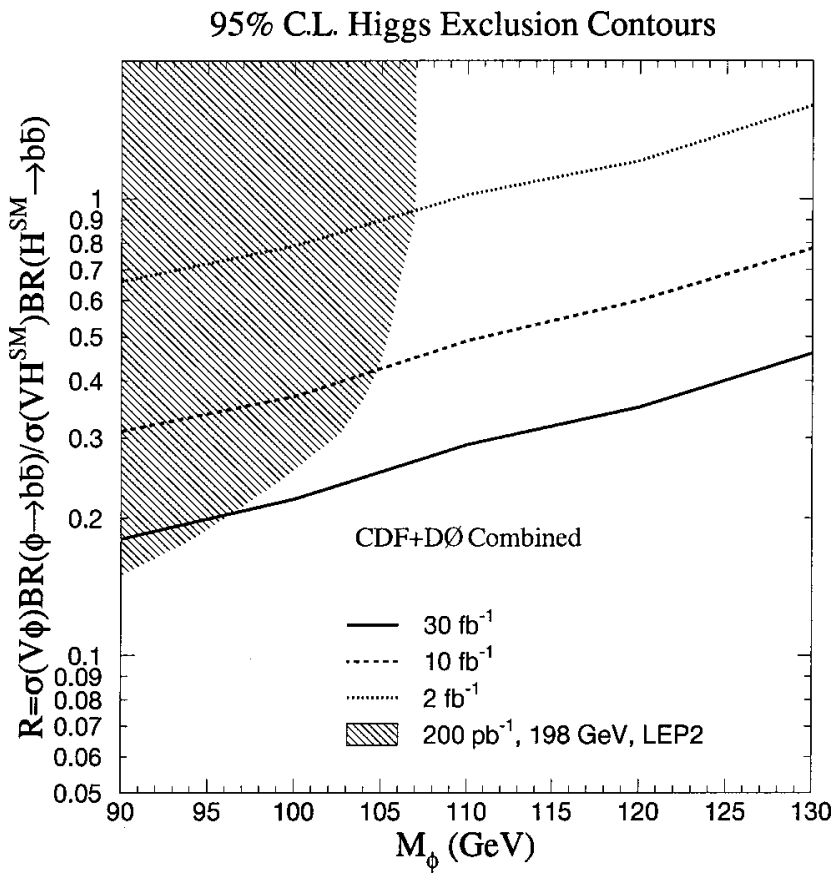

FIG. 1. $95 \%$ C.L. bound on $R=[\sigma(p \bar{p} \rightarrow V \phi) / \sigma(p \bar{p}$ $\left.\left.\rightarrow V H^{\mathrm{SM}}\right)\right]\left[B(\phi \rightarrow b \bar{b}) / B\left(H^{\mathrm{SM}} \rightarrow b \bar{b}\right)\right]$ (with $V=W$ or $Z$ ) as a function of the Higgs boson mass for the Tevatron and LEP2.

$\left(m_{A}, \tan \beta\right)$ can be excluded or discovered. The numerical results are illustrated in Figs. 3-6. Figures 3-5 correspond to three common choices of the MSSM parameters. This set is not exhaustive, but is meant to illustrate the effect of the top and bottom squark mixing parameters when all sparticles are relatively heavy. We have chosen the squarks masses to have the value $M_{S}=1 \mathrm{TeV}$ and the Higgsino mass parameter to

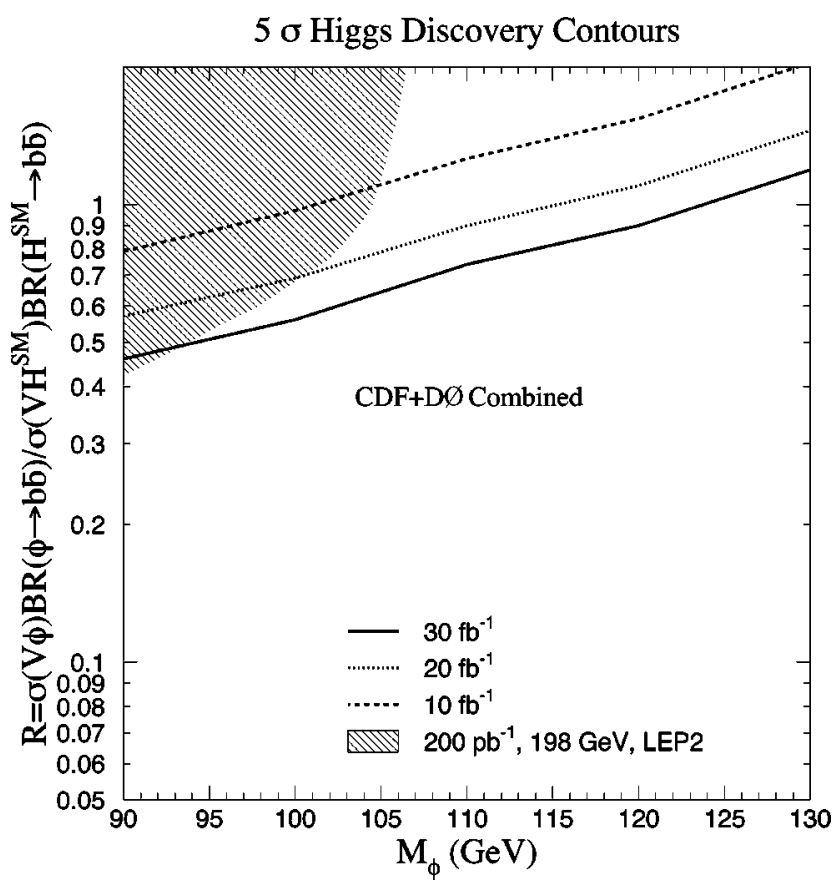

FIG. 2. Same as Fig. 1 except for $5 \sigma$ discovery. 


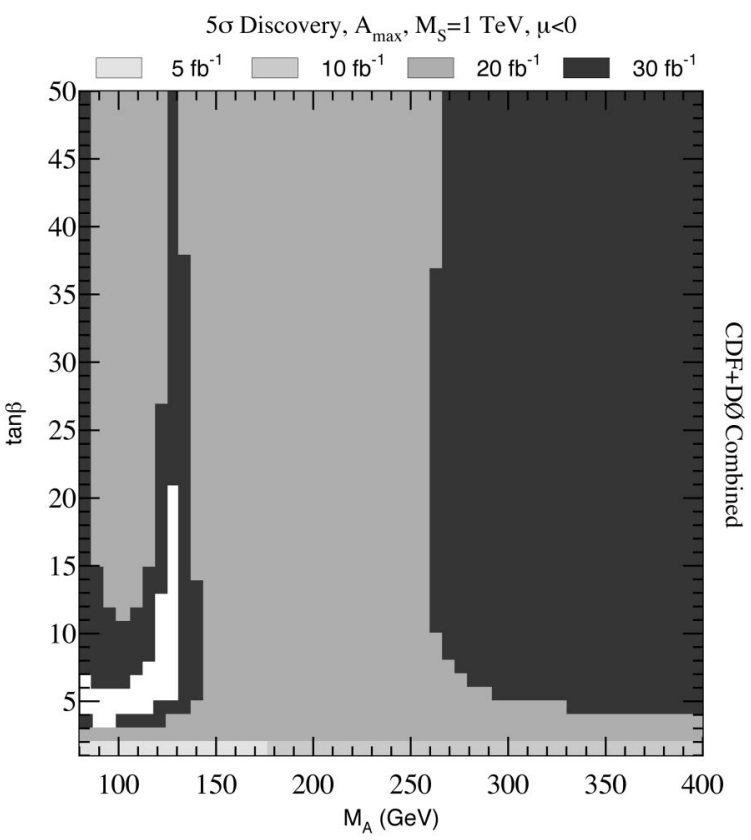

FIG. 3. Projected $5 \sigma$ discovery contours for the $V \phi(\rightarrow b \bar{b})$ mode $(V=W$ or $Z)$ at the Tevatron in the MSSM for maximal mixing, $\mu<0$, and $M_{S}=1 \mathrm{TeV}$. The results assume a combination of $\mathrm{CDF}$ and $\mathrm{D} \varnothing$ data, a $10 \% m_{b \bar{b}}$ mass resolution, and systematic errors as explained in the text. Different shadings correspond to different integrated luminosities.

have the values $\pm 1 \mathrm{TeV}$. The top squark trilinear coupling $A_{t}$ is chosen either to make the top squark mixing parameter very small (minimal mixing), $\tilde{a} \simeq 0$, or, in the limit of large $m_{A}$, to maximize the lightest $C P$-even Higgs mass (maximal mixing), $|\tilde{a}| \simeq \sqrt{6}$. Four integrated luminosities are assumed: $5,10,20$, and $30 \mathrm{fb}^{-1}$.

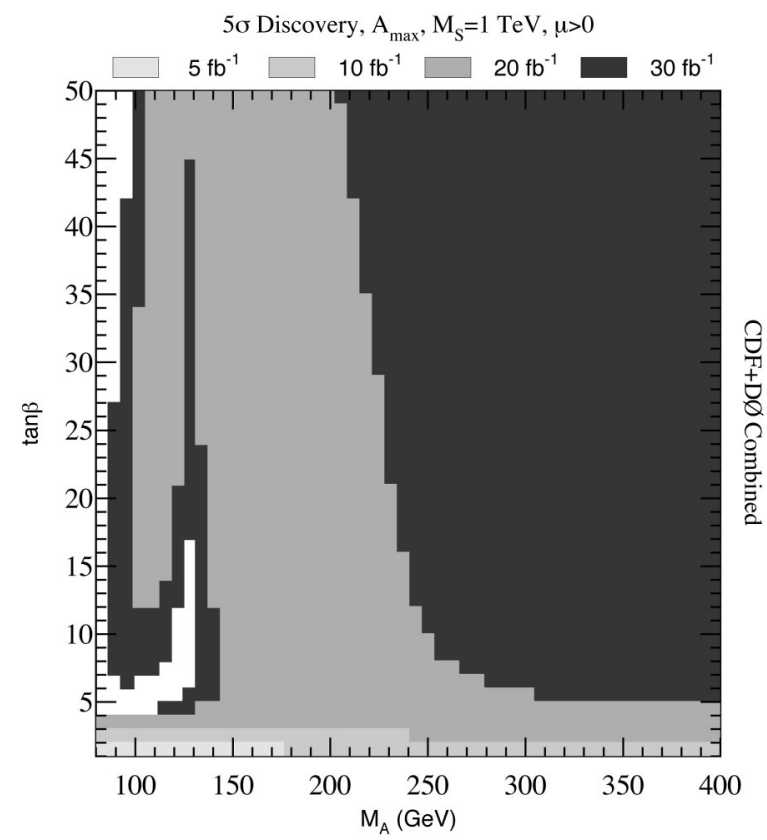

FIG. 4. Same as Fig. 3 but with $\mu>0$.

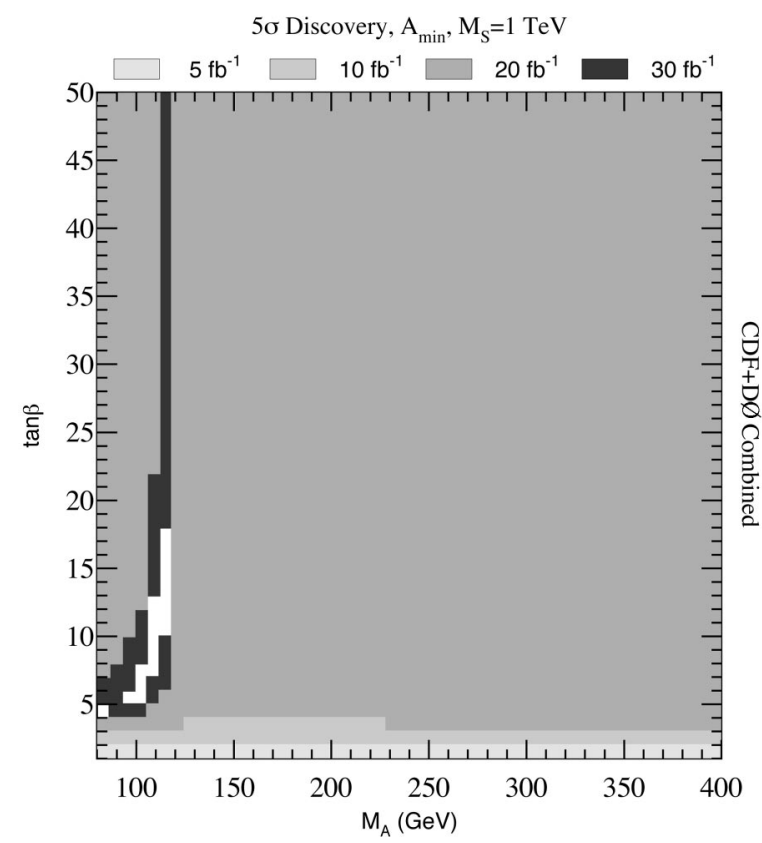

FIG. 5. Same as Fig. 3 but for minimal mixing.

In the case of maximal mixing, the radiative corrections to the $\mathcal{M}_{12}^{2}$ depend on the sign of $\bar{\mu} \times \tilde{a}$. This dependence is obvious in Figs. 3 and 4, where the discovery reach for the cases $\mu= \pm 1 \mathrm{TeV}$ and $\tilde{a}=\sqrt{6}$ is displayed. An increase or decrease of the effective Yukawa coupling of a standard model-like Higgs boson to bottom quarks, for negative or positive values of $\bar{\mu} \times \tilde{a}$, leads to large variations in the branching ratio to $b$ quarks. This has an important impact on the luminosity required to observe a Higgs boson in this channel. For Higgs boson masses in the range $125-130 \mathrm{GeV}$, as expected for the SM-like Higgs boson in the limit of large

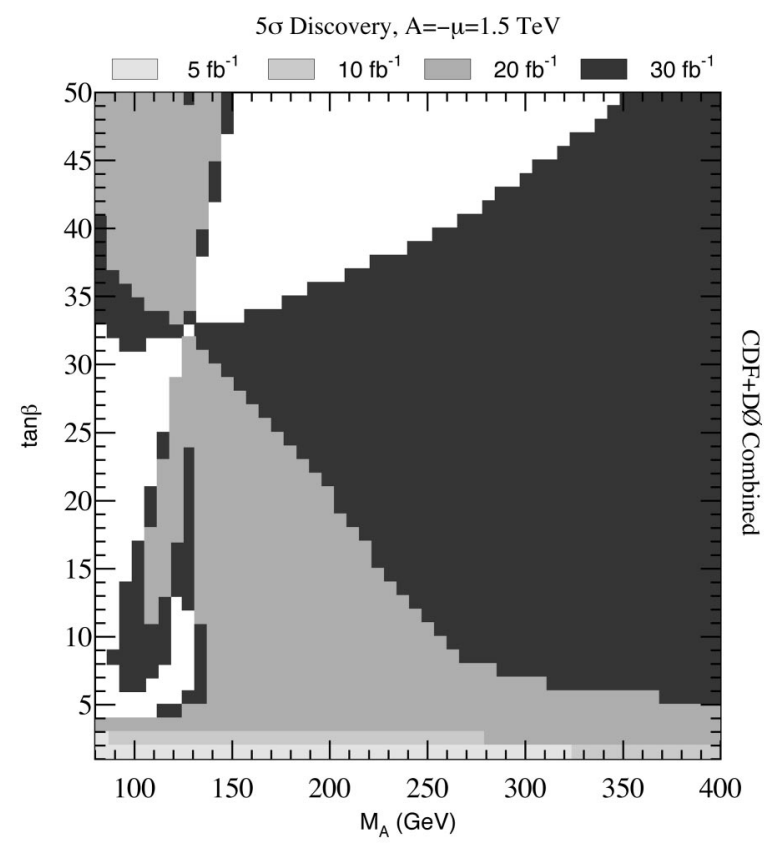

FIG. 6. Same as Fig. 3, but for $A=-\mu=1.5 \mathrm{TeV}$. 
$\tan \beta$ and maximal mixing, a decrease in the branching ratio to bottom quarks is compensated by an increase in the branching ratio to $W^{(*)} W^{(*)}$.

Figures 3 and 4 demonstrate that the dependence on the sign of $\bar{\mu} \times \tilde{a}$ is particularly strong for low values of $m_{A}$, but, as we discussed before, might become relevant even for relatively large values of $m_{A} \simeq \mathcal{O}(300 \mathrm{GeV})$. The suppression of the coupling which is obtained for low values of $m_{A}$ and large values of $\tan \beta$ leads to a problem for detecting the heavy Higgs boson for positive values of $\bar{\mu} \times \tilde{a}$ [see Eq. (2.5)].

The region of $m_{A} \simeq 120 \mathrm{GeV}\left(m_{A} \simeq m_{h}\right)$ and large $\tan \beta$ is difficult to observe, since this is the region of maximal mixing and $\sin ^{2}(\beta-\alpha) \simeq \cos ^{2}(\beta-\alpha) \simeq \mathcal{O}(0.5)$. Although this limitation can be overcome with more luminosity, a window of nonobservability would remain for both signs of $\bar{\mu} \times \tilde{a}$. Fortunately, if $\tan \beta$ is sufficiently large, the two $C P$-even Higgs bosons tend to have similar masses. Whenever the mass difference is less than $10 \mathrm{GeV}$, we have added the individual significances of the two signals in quadrature. Using this procedure, we can extend the coverage for very large $\tan \beta$ and $m_{A} \simeq m_{h}$. However, a window of nonobservability remains for $\tan \beta \simeq 5$, since the mass difference is large and $\sin (\beta-\alpha)$ is suppressed in this region. ${ }^{7}$ Finally, the region of $m_{A} \simeq 150 \mathrm{GeV}$ is easier to observe, since $\sin ^{2}(\beta-\alpha)$ is already of order one in this region and the bottom coupling of the lightest $C P$-even Higgs boson is strongly enhanced with respect to the standard model case, implying an increase in the branching ratio of this Higgs boson into bottom quarks.

In Fig. 5, the case of minimal mixing $(\tilde{a}=0, \bar{\mu}=1)$ is displayed. In the large $m_{A}$ limit, the lightest $C P$-even Higgs boson mass is of order $110-115 \mathrm{GeV}$ for moderate or large values of $\tan \beta$ and hence detectable for luminosities of order $10 \mathrm{fb}^{-1}$. The characteristics of this case are similar to the case of maximal mixing, although, due to the lower values of the lightest $C P$-even Higgs mass, lower luminosities are required to cover the large $m_{A}$ region and, in addition, the window of nonobservability for $30 \mathrm{fb}^{-1}$ shrinks to a very small region of parameter space for $m_{A} \simeq m_{h}$. For minimal mixing, the results are insensitive to the sign of $\mu$. This occurs since the dependence of the Higgs boson mass matrix (and hence of the $C P$-even Higgs masses and their couplings to bottom quarks) on the sign of $\mu$ arises through the radiative corrections to the off-diagonal elements, which are proportional to $\bar{\mu} \times \tilde{a}$ and vanish when $\tilde{a} \simeq 0$.

In Fig. 6, we present the case in which the stop mixing parameters are such that the bottom Yukawa coupling of the standard model-like Higgs boson can be efficiently suppressed in a large region of parameters. For this, we have taken values of the mixing parameters $A_{t}=-\mu=1.5 \mathrm{TeV}$. In this case, in the limit of large values of $m_{A}$ and for moderate or large values of $\tan \beta$, the lightest $C P$-even Higgs

\footnotetext{
${ }^{7}$ If we had not combined the signals, the region of nonobservability for $m_{A} \simeq m_{h}$ would have extended to $\tan \beta \simeq 50$ (30) in Fig. 3 (4).
}

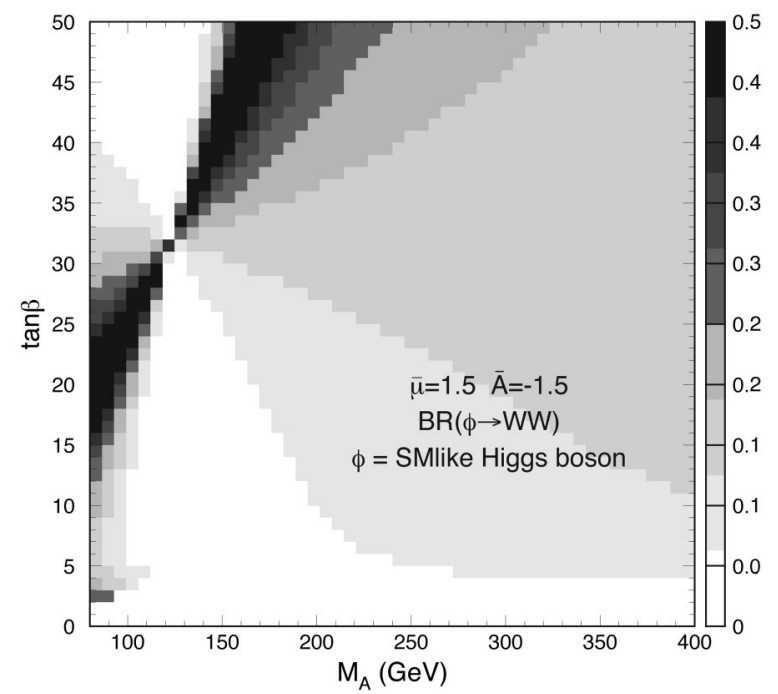

FIG. 7. The expected variation of the branching ratio for Higgs boson decays to $W^{*} W^{*}$ for the MSSM parameters of Fig. 6 .

boson mass takes values in the range $120-125 \mathrm{GeV}$. Windows of nonobservability appear associated with the suppression of the bottom Yukawa coupling of the standard modellike Higgs boson, i.e., vanishing $\mathcal{M}_{12}$. For large values of $\tan \beta$ the window of nonobservability extends up to relatively large values of the $C P$-odd Higgs mass. It would be very interesting to check if these windows may be efficiently covered by using the $W W$ decay mode of the Higgs boson $[15,20]$. Figure 7 shows the expected variation of the branching ratio for Higgs boson decays to $W^{*} W^{*}$ for this choice of MSSM parameters, and values near 50\% are possible.

It is clear from Figs. 3-6 that a single detector at the Tevatron requires about $30 \mathrm{fb}^{-1}$ for a reasonable coverage of the MSSM parameter space, far beyond the region already covered by LEP2. Other decay channels besides $h, H \rightarrow b \bar{b}$ need to be explored to cover specific regions of parameter space. For values of $M_{S} \simeq 1 \mathrm{TeV}$ and moderate or large values of $\tan \beta$, the standard model-like Higgs boson tends to be heavier than $100 \mathrm{GeV}$. In this case, the LEP reach in the $Z h$ and $Z H$ channels is highly reduced, and most of the coverage usually shown is induced by the $h A$ production (since $H A$ is kinematically limited). ${ }^{8}$ An essential advantage of the Tevatron is the fact that it can overcome this kinematic limitation and give a significant coverage of the $m_{A}$-tan $\beta$ plane via the $V h$ and $V H$ channels even for large values of $\tan \beta$.

\section{YUKAWA COUPLING EFFECTS IN THE LARGE $\tan \beta$ REGIME}

In the SM, the coupling of the Higgs boson to $b$ quarks is proportional to the bottom Yukawa coupling $h_{b}^{\mathrm{SM}} \equiv m_{b} / \mathrm{v}$. Within the MSSM, the effective bottom Yukawa coupling can be quite different than in the standard model case. This is

\footnotetext{
${ }^{8}$ For $\sqrt{s}=200 \mathrm{GeV}$ and $200 \mathrm{pb}^{-1}$, LEP2 can discover a Higgs boson in the $h A$ channel for $m_{A}<90 \mathrm{GeV}$ and large $\tan \beta$.
} 
due not only to the dependence on the Higgs mixing angles, discussed above, but also to the presence of large radiative corrections in the coupling of bottom quarks and $\tau$ leptons to the neutral components of the Higgs doublets, that lead to modifications of the relation, Eq. (1.1), between the bottom Yukawa coupling and the running bottom mass [21-23]. To better understand this, it is necessary to concentrate on the properties of the large $\tan \beta$ regime. In this regime, to a first approximation, only $H_{2}$ acquires a vacuum expectation value [here $\mathrm{H}_{2}$ denotes the whole $S U(2)_{L}$ doublet]. This means that this Higgs doublet contains the three Goldstone bosons and a neutral Higgs boson with standard model-like couplings to the electroweak gauge bosons. The other Higgs doublet $H_{1}$ does not communicate with the electroweak symmetry breaking sector and contains, in a first approximation, a $C P$-even and a $C P$-odd Higgs field, which are almost degenerate in mass, and a charged Higgs field, whose mass differs from $m_{A}$ only in a $D$ term $m_{H^{ \pm}}^{2}=m_{A}^{2}+M_{W}^{2}$. The SMlike Higgs boson acquires a mass given by

$$
m_{\phi^{S \mathrm{SM}}}^{2} \simeq \mathcal{M}_{22}^{2} \simeq M_{Z}^{2}+\text { radiative corrections, }
$$

and its dependence on $m_{A}$ is suppressed by a $1 / \tan ^{2} \beta$ factor [see Eqs. (2.2) and (2.3)].

Supersymmetric one-loop corrections to the tree-level, running bottom quark mass can be significant for large values of $\tan \beta$ and translate directly into a redefinition of the relation between the bottom Yukawa coupling entering in the production and decay processes and the physical (pole) bottom mass. Some of the phenomenological implications of these corrections have been considered for MSSM Higgs boson decays [24]. The main reason why these one-loop corrections are particularly important is that they do not decouple in the limit of a heavy supersymmetric spectrum. As mentioned above, in the supersymmetric limit, bottom quarks only couple to the neutral Higgs field $H_{1}^{0}$. However, supersymmetry is broken and the bottom quark will receive a small coupling to the Higgs field $H_{2}^{0}$ from radiative corrections,

$$
\mathcal{L} \simeq h_{b} H_{1}^{0} b \bar{b}+\Delta h_{b} H_{2}^{0} b \bar{b} .
$$

The coupling $\Delta h_{b}$ is suppressed by a small loop factor compared to $h_{b}$ and hence, one would be inclined to neglect it. ${ }^{9}$ However, once the Higgs doublet acquires a vacuum expectation value, the running bottom mass receives contributions proportional to $\Delta h_{b} \vee_{2}$. Although $\Delta h_{b}$ is one-loop suppressed with respect to $h_{b}$, for sufficiently large values of $\tan \beta \quad\left(\mathrm{v}_{2} \gg \mathrm{v}_{1}\right)$ the contribution to the bottom quark mass of both terms in Eq. (3.2) may be comparable in size. This induces a large modification in the tree level relation, Eq. (1.1),

$$
m_{b}=h_{b} \mathrm{v}_{1}\left[1+\Delta\left(m_{b}\right)\right]
$$

\footnotetext{
${ }^{9}$ In the above we are explicitly neglecting corrections to the $h_{b}$ coupling of $\mathcal{O}\left(\Delta h_{b}\right)$.
}

where $\Delta\left(m_{b}\right)=\Delta h_{b} \tan \beta / h_{b}$.

The function $\Delta\left(m_{b}\right)$ contains two main contributions, one from a bottom squark-gluino loop (depending on the two bottom squark masses $M_{\widetilde{b}_{1}}$ and $M_{\tilde{b}_{2}}$ and the gluino mass $M_{\tilde{g}}$ ) and another one from a top-squark-Higgsino loop (depending on the two top squark masses $M_{\tilde{t}_{1}}$ and $M_{\tilde{t}_{2}}$ and the Higgsino mass parameter $\mu)$. The explicit form of $\Delta\left(m_{b}\right)$ at one-loop can be approximated by computing the supersymmetric loop diagrams at zero external momentum $\left(M_{S}\right.$ $\left.\gg m_{b}\right)$ and is given by [21-23]

$$
\begin{aligned}
\Delta\left(m_{b}\right) \simeq & \frac{2 \alpha_{3}}{3 \pi} M_{\tilde{g}} \mu \tan \beta I\left(M_{\tilde{b}_{1}}, M_{\tilde{b}_{2}}, M_{\tilde{g}}\right) \\
& +\frac{Y_{t}}{4 \pi} A_{t} \mu \tan \beta I\left(M_{\tilde{t}_{1}}, M_{\tilde{t}_{2}}, \mu\right),
\end{aligned}
$$

where $\alpha_{3}=g_{3}^{2} / 4 \pi, \quad Y_{t}=h_{t}^{2} / 4 \pi$, and the function $I$ is given by

$I(a, b, c)=\frac{a^{2} b^{2} \ln \left(a^{2} / b^{2}\right)+b^{2} c^{2} \ln \left(b^{2} / c^{2}\right)+c^{2} a^{2} \ln \left(c^{2} / a^{2}\right)}{\left(a^{2}-b^{2}\right)\left(b^{2}-c^{2}\right)\left(a^{2}-c^{2}\right)}$,

and is positive by definition. Smaller contributions to $\Delta\left(m_{b}\right)$ have been neglected for the purpose of this discussion. It is important to remark that these effects are just a manifestation of the lack of supersymmetry in the low energy theory and, hence, $\Delta\left(m_{b}\right)$ does not decouple in the limit of large values of the supersymmetry breaking masses. Indeed, if all supersymmetry breaking parameters (and $\mu$ ) are scaled by a common factor, the correction $\Delta\left(m_{b}\right)$ remains constant.

Similarly to the bottom case, the relation between $m_{\tau}$ and the $\tau$ lepton Yukawa coupling $h_{\tau}$ is modified:

$$
m_{\tau}=h_{\tau} \vee_{1}\left[1+\Delta\left(m_{\tau}\right)\right]
$$

The function $\Delta\left(m_{\tau}\right)$ contains a contribution from a tau slepton-bino loop (depending on the two stau masses $M_{\tau_{1}}$ and $M_{\tilde{\tau}_{2}}$ and the bino mass parameter $M_{1}$ ) and a tau sneutrino-chargino loop (depending on the tau sneutrino mass $M_{\tilde{\nu}_{\tau}}$, the wino mass parameter $M_{2}$ and $\mu$ ). It is given by the expression $[22,23]$

$$
\begin{aligned}
\Delta\left(m_{\tau}\right)= & \frac{\alpha_{1}}{4 \pi} M_{1} \mu \tan \beta I\left(M_{\tilde{\tau}_{1}}, M_{\tilde{\tau}_{2}}, M_{1}\right) \\
& +\frac{\alpha_{2}}{4 \pi} M_{2} \mu \tan \beta I\left(M_{\tilde{\nu}_{\tau}}, M_{2}, \mu\right),
\end{aligned}
$$

where $\alpha_{1}=g_{1}^{2} / 4 \pi, g_{1}$ is the $U(1)$ hypercharge coupling, $\alpha_{2}=g_{2}^{2} / 4 \pi, g_{2}$ is the $S U(2)$ weak isosopin coupling.

Since corrections to $h_{\tau}$ are proportional to $\alpha_{1}$ and $\alpha_{2}$, they are expected to be smaller than the corrections to $h_{b}$. Although the precise values of $\Delta\left(m_{b}\right)$ and $\Delta\left(m_{\tau}\right)$ are model dependent, the leading term in the tau mass corrections has a factor $M_{2} \mu I\left(M_{\tilde{\nu}_{\tau}}, M_{2}, \mu\right) \propto M_{2} \mu / \max \left(m_{\tilde{\nu}_{\tau}}^{2}, M_{2}^{2}, \mu^{2}\right) \leqslant 1$, and hence, for $\tan \beta \leqq 50, \Delta\left(m_{\tau}\right)<0.15$. In the following, we 
consider the impact of the bottom mass corrections assuming $\Delta\left(m_{\tau}\right) \ll \Delta\left(m_{b}\right)$, and using the expression $\Delta\left(m_{b}\right)=K \tan \beta$ to parametrize possible radiative corrections. Since the value of $\alpha_{3}$ at the scale $M_{S}$ is of order 0.1, and if all soft supersymmetry breaking parameters and $\mu$ are of order of $1 \mathrm{TeV}$, the coefficient $K$ can have either sign and will be of order $|K| \simeq 0.01$. One can also consider cases in which the bottom mass corrections are highly suppressed. This happens naturally in the case of approximate $R$ and Peccei-Quinn symmetries in the theory, which make the value of the gaugino masses and the top squark mixing parameters much lower than $M_{S}[21]$.

It is instructive to return to the couplings of the lightest and heaviest $C P$-even Higgs bosons and of the $C P$-odd Higgs boson to bottom quarks. The $C P$-odd Higgs boson coupling to bottom quarks is given by

$$
\mathcal{L}=-i h_{b}^{C P} A \bar{b} \gamma_{5} b
$$

with

$$
h_{b}^{C P}=h_{b} \sin \beta+\Delta h_{b} \cos \beta \simeq h_{b} \sin \beta=\frac{m_{b}}{\left[1+\Delta\left(m_{b}\right)\right] \mathrm{v}} \tan \beta .
$$

Using Eqs. (3.2) and (1.2), together with the relation of the bottom Yukawa coupling to the bottom mass, Eq. (3.3), it is easy to show that the effective couplings of the $C P$-even Higgs bosons $\bar{h}_{b}$ and $\widetilde{h}_{b}$

$$
\mathcal{L}=\bar{h}_{b} b \bar{b} h+\widetilde{h}_{b} b \bar{b} H
$$

are approximately given by

$$
\begin{gathered}
\bar{h}_{b} \simeq-\frac{m_{b} \sin \alpha}{\mathrm{v} \cos \beta}\left[1-\frac{\Delta\left(m_{b}\right)}{1+\Delta\left(m_{b}\right)}\left(1+\frac{1}{\tan \alpha \tan \beta}\right)\right], \\
\widetilde{h}_{b} \simeq \frac{m_{b} \cos \alpha}{\mathrm{V} \cos \beta}\left[1-\frac{\Delta\left(m_{b}\right)}{1+\Delta\left(m_{b}\right)}\left(1-\frac{\tan \alpha}{\tan \beta}\right)\right] .
\end{gathered}
$$

The value of $\Delta\left(m_{b}\right)$ in Eq. (3.4) is defined at the scale $M_{S}$, where the sparticles are decoupled. The $h_{b}$ and $\Delta h_{b}$ couplings should be computed at that scale, and run down with their respective renormalization group equations to the scale $m_{A}$, where the relations between the couplings of the bottom quark to the neutral Higgs bosons and the running bottom quark mass, Eqs. (3.9), (3.11), and (3.12) are defined. In the present study we have defined the running bottom mass at the scale $m_{A}$ as a function of $m_{b}\left(m_{b}\right) \simeq 4.25 \mathrm{GeV}$ while using two-loop renormalization group equations in the effective standard model theory at scales $Q<m_{A}$. The above procedure leads to a consistent definition of the bottom quark couplings to the Higgs bosons when the three neutral Higgs boson masses are of the same order. For large values of the $C P$-odd Higgs boson mass, instead, $\bar{h}_{b}$ must be evolved with SM renormalization group equations from $m_{A}$ down to $m_{h}$. The definition of the couplings of the bottom quark to the Higgs bosons at the scale of the corresponding Higgs boson mass is chosen to take into account the bulk of the QCD correction. Indeed, it is known that this choice of scale represents well the bulk of the QCD corrections to the Higgs boson decay into quarks and gluons [1]. However, for the production process $b \bar{b} \phi$, a complete study of the NLO effects remains necessary to get a definitive estimate of the Tevatron reach in this production channel.

It is interesting to study different limits of the above couplings. For $m_{A} \gg m_{\phi} \mathrm{sM}$, the lightest $C P$-even Higgs boson should behave similar to the SM particle. Indeed, in this limit $\cos \beta \simeq-\sin \alpha$ and $\sin \beta \simeq \cos \alpha$, and $\bar{h}_{b}=m_{b} / \mathrm{v}$, which is the standard coupling. In the same limit, $\tilde{h}_{b} \simeq h_{b} \sin \beta\{1$ $\left.+\mathcal{O}\left[\Delta\left(m_{b}\right) / \tan ^{2} \beta\right]\right\}$. Even in the presence of radiative corrections to the bottom quark couplings, the heaviest $C P$-even Higgs boson coupling is approximately equal to the $C P$-odd one. When $m_{A}$ approaches $m_{\phi}$ sM, the above relations between the angles $\alpha$ and $\beta$ are slightly violated. Due to the large $1 / \cos \beta$ factor appearing in the definition of the Yukawa coupling, Eq. (3.11), a small departure from the above relations can induce large departures of the coupling $\bar{h}_{b}$ with respect to the standard model value. For $m_{A}$ $\ll m_{\phi} \mathrm{sm}$, instead, $\sin \beta \simeq-\sin \alpha \simeq 1$. The lightest Higgs boson coupling is $\bar{h}_{b} \simeq h_{b} \sin \beta\left[1+\mathcal{O}\left(\Delta m_{b} / \tan ^{2} \beta\right)\right]$, while, as happens for vanishing values of the bottom mass corrections, the coupling of the heaviest $C P$-even Higgs boson may become highly nonstandard.

As discussed in Sec. II, in the large $\tan \beta$ regime, the off-diagonal elements of the mass matrix can receive large radiative corrections with respect to the tree-level value, $\left(\mathcal{M}_{12}^{2}\right)^{\text {tree }} \simeq-\left(m_{A}^{2}+M_{Z}^{2}\right) / \tan \beta$. When both $\Delta\left(m_{b}\right)$ and $\Delta\left(m_{\tau}\right)$ are small, the coupling of the standard model-like Higgs boson to bottom quarks and $\tau$ leptons vanishes for vanishing $\sin 2 \alpha$ [see Eq. (2.4)]. The reason for this cancellation when $\sin 2 \alpha=0$ is that the standard model-like Higgs boson becomes a pure $H_{2}^{0}$ state, which does not couple to bottom quarks and $\tau$ leptons at tree level. If the bottom and $\tau$ mass corrections are large, however, the bottom and $\tau$ couplings do not cancel for $\sin 2 \alpha=0$, but are just given by $\Delta h_{b}$ and $\Delta h_{\tau}$, respectively. Indeed, from Eq. (3.11) [Eq. (3.12)], in the limit $\sin \alpha=0 \quad(\cos \alpha=0)$, the bottom coupling is given by

$$
\bar{h}_{b}\left(\widetilde{h}_{b}\right)=\frac{m_{b}}{\sin \beta v} \times \frac{\Delta\left(m_{b}\right)}{\left[1+\Delta\left(m_{b}\right)\right]} \equiv \Delta h_{b} .
$$

In this limit, the coupling to bottom quarks is much smaller than the standard model coupling only if $\left|\Delta\left(m_{b}\right)\right| \ll 1$. A similar expression to Eq. (3.13) holds for the $\tau$ lepton coupling.

For values of $\Delta\left(m_{b}\right)$ of order 1 , however, a strong suppression of the bottom coupling $\bar{h}_{b}$ can still occur for slightly different values of the Higgs mixing angle $\alpha$, namely,

$$
\tan \alpha \simeq \frac{\Delta\left(m_{b}\right)}{\tan \beta} .
$$

Under these conditions, 


$$
\bar{h}_{\tau}=\frac{m_{\tau}}{\mathrm{v} \sin \beta}\left(\frac{\Delta\left(m_{\tau}\right)-\Delta\left(m_{b}\right)}{1+\Delta\left(m_{\tau}\right)}\right), \quad \bar{h}_{b}=0
$$

A similar expression is obtained for the coupling $\widetilde{h}_{\tau}$ in the case $\tilde{h}_{b}=0$. Hence, if $\tan \beta$ is very large and $\Delta\left(m_{b}\right)$ is of order one, the $\tau$ Yukawa coupling may not be strongly suppressed with respect to the standard model case and can provide the dominant decay mode for a standard model-like Higgs boson.

To recapitulate, the cancellation in the off-diagonal elements of the mass matrix can lead to a strong suppression of the standard model-like Higgs boson coupling to bottom quarks and $\tau$ leptons. In general, this implies a sharp increase of the branching ratio of the decay of this Higgs into gauge bosons and charm quarks. However, for very large values of $\tan \beta$ and values of the bottom mass corrections $\Delta\left(m_{b}\right)$ of order 1 , the branching ratio of the decay into $\tau$ leptons may increase in the regions in which the bottom quark decays are strongly suppressed.

\section{HIGGS BOSON PHENOMENOLOGY WITH LARGE $\tan \beta$ CORRECTIONS}

\section{A. $V \phi$ channel}

The finite corrections to the bottom Yukawa coupling are important in defining the exact regions for which the bottom Yukawa coupling is suppressed. Depending on the sign of the bottom mass corrections and on the specific region of supersymmetric parameter space, important increases or decreases in coverage may occur with respect to the case of $\Delta\left(m_{b}\right)=0$. For large $m_{A}$, the coupling of the lightest $C P$-even Higgs boson is only slightly affected by the presence of $\Delta\left(m_{b}\right)$, and these corrections will not affect the discovery potential. The only exception is when the negative contributions to the $\mathcal{M}_{22}^{2}$ matrix elements, proportional to $h_{b}^{4}$, become relevant [see Eq. (2.3)]. For low values of $m_{A}$, instead, the bottom mass corrections might have an important impact in the discovery and exclusion reach for a given choice of parameters.

Figures 8-11 show the impact of the bottom mass corrections on the discovery reach of the $C P$-even Higgs bosons in the $V \phi$ channel for the case of maximal mixing $\mu>0$ and both signs of the bottom mass corrections, assumed to be given by $\Delta m_{b}=K \times \tan \beta$, with $K= \pm 0.05$ and \pm 0.01 . Since the Higgs sector parameters depend only on the size of the mixing parameters and on the sign of $\bar{\mu} \times \bar{A}_{t}$, while the bottom mass corrections depend also on the sign of $\bar{\mu} \times M_{\tilde{g}}$, one can have either sign for the bottom mass corrections, for fixed values of the top squark mixing parameters. Although the most generic features of the discovery reach plots are not changed by the presence of the bottom mass corrections, positive bottom mass corrections tend to reduce the bottom Yukawa couplings and increase the luminosity needed for a Higgs boson discovery. The opposite happens in the presence of negative mass corrections. Observe that, for values of $K=-0.01$, there is an improvement of the discovery reach at very large values of $\tan \beta$. This improvement is

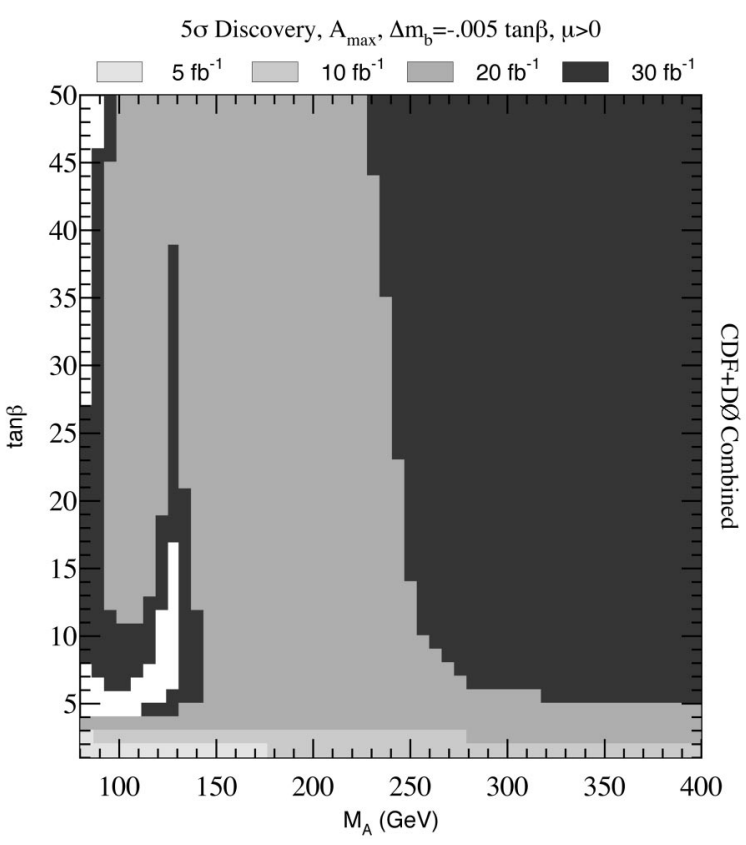

FIG. 8. Same as Fig. 3 for maximal mixing and $\mu>0$ after including large $\tan \beta$ corrections: $\Delta\left(m_{b}\right)=-0.005 \tan \beta$.

related to a decrease in the lightest $C P$-even Higgs boson mass induced by the negative corrections to the $\mathcal{M}_{22}^{2}$ Higgs mass squared matrix elements, proportional to $h_{b}^{4}$, which become enhanced for large values of $\tan \beta$ and negative values of $K$. As we shall discuss below, in minimal supersymmetry breaking models, the bottom mass corrections tend to be positive, and hence the reach of the Tevatron is negatively affected. The same plots for the case of minimal mixing do not show as much sensitivity.

The reason why positive bottom mass corrections suppress the reach of the Tevatron collider can be easily under-

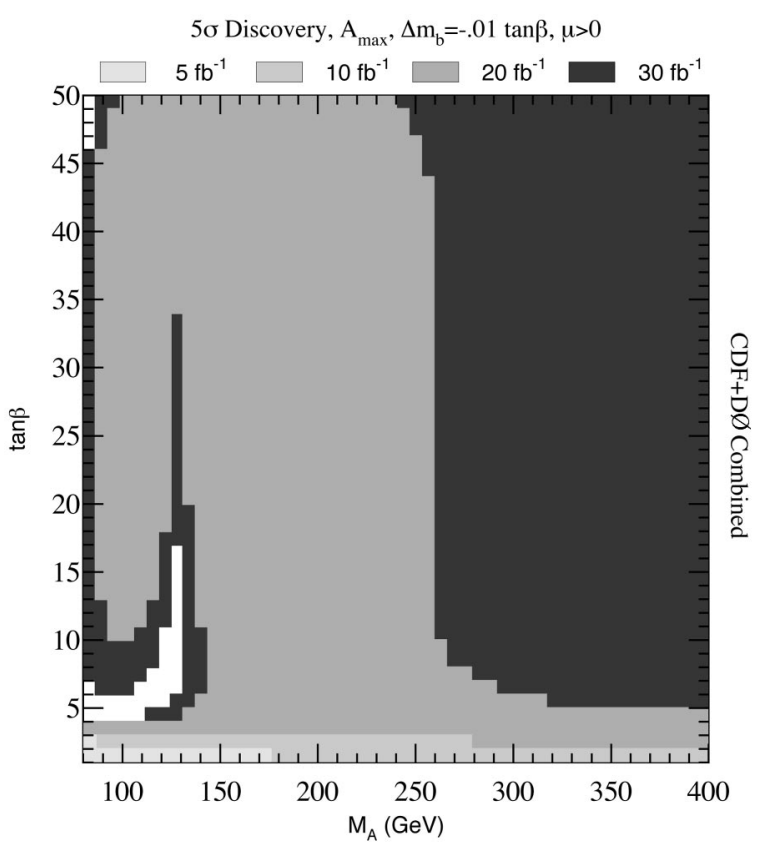

FIG. 9. Same as Fig. 8 but for $\Delta\left(m_{b}\right)=-0.01 \tan \beta$. 


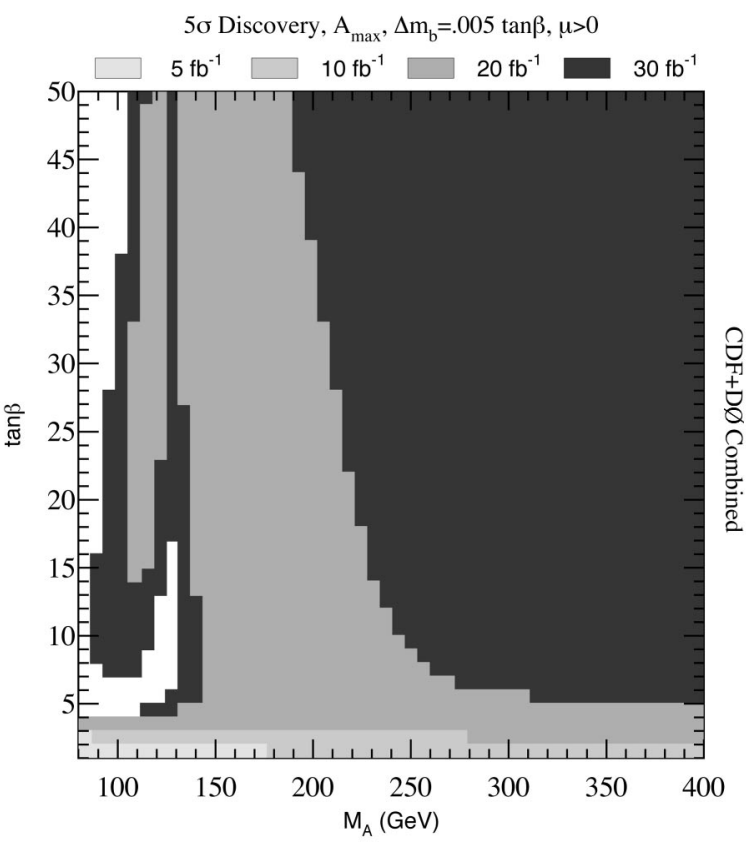

FIG. 10. Same as Fig. 8 but for $\Delta\left(m_{b}\right)=0.005 \tan \beta$.

stood by studying the behavior of the effective bottom Yukawa couplings, Eqs. (3.11) and (3.12). Indeed, for $m_{A}$ $\ll m_{\phi}$ SM, since $\tan \alpha / \tan \beta<0$, the expression between parentheses in Eq. (3.12) is positive. For a fixed value of the angles $\alpha$ and $\beta$, a positive $\Delta\left(m_{b}\right)$ tends to reduce the value of $\tilde{h}_{b}$. For $m_{A} \gg m_{\phi} \mathrm{sM}$, instead, since $\tan \alpha \times \tan \beta<0$, the effect of the bottom mass corrections on the value of $\bar{h}_{b}$, Eq. (3.11), depends on whether $|\tan \alpha \times \tan \beta|$ is greater or less than 1. In the cases displayed in Figs. 9-12, this factor is always larger than one and a positive bottom mass correction reduces the value of $\bar{h}_{b}$.

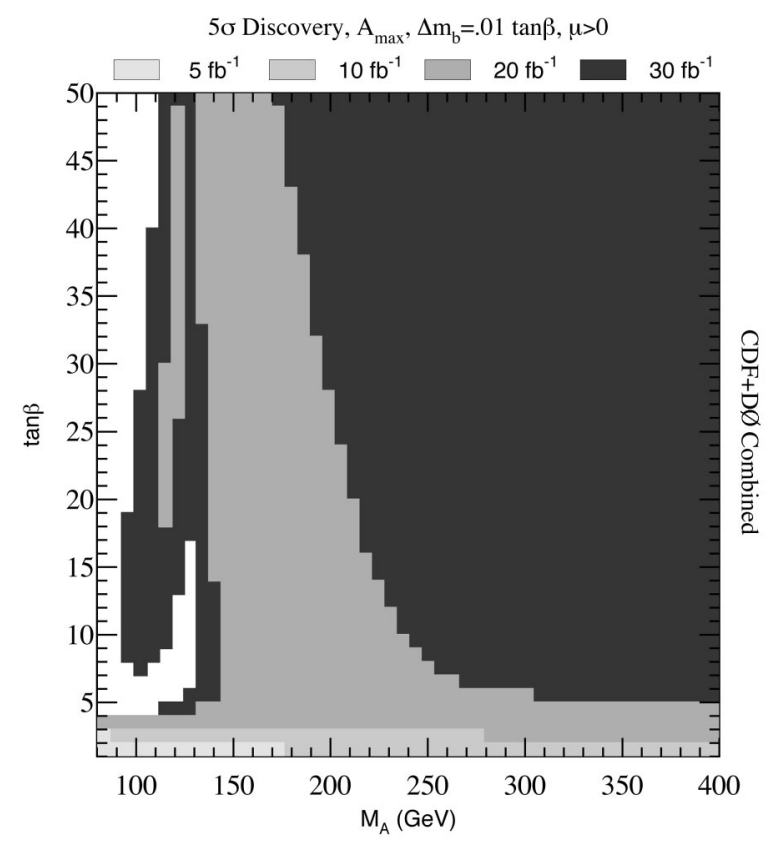

FIG. 11. Same as Fig. 8 but for $\Delta\left(m_{b}\right)=0.01 \tan \beta$.

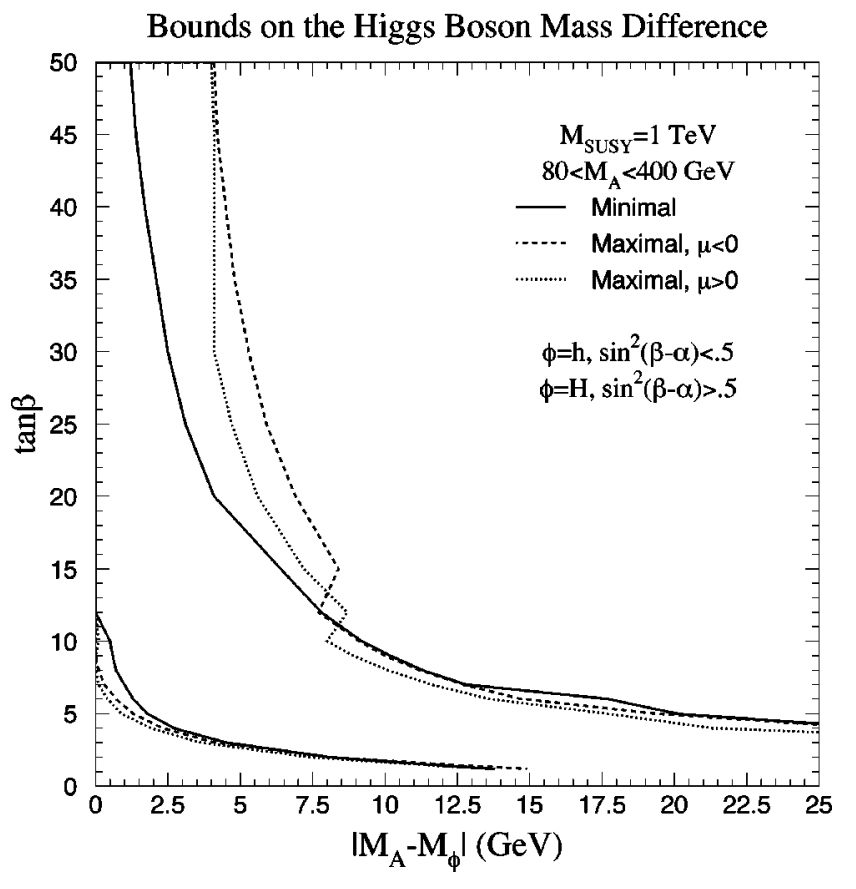

FIG. 12. Mass difference between the $C P$-odd Higgs boson and the $C P$-even Higgs boson (with properties similar to the $C P$-odd one) as a function of $\tan \beta$ for several MSSM parameter choices.

\section{B. $b \bar{b} \phi$ channel}

The Yukawa coupling corrections discussed above affect the associated production of a Higgs boson with $b$ quarks, where the Higgs boson subsequently decays to a heavy flavor final state. Indeed, the cross section times branching ratio for this process at an $h_{1} h_{2}$ hadron collider satisfies

$$
\sigma\left[h_{1} h_{2} \rightarrow b \bar{b} \phi(\rightarrow b \bar{b})+X\right] \propto \hat{h}_{b}^{2} \times \hat{B}_{b}
$$

and

$$
\sigma\left[h_{1} h_{2} \rightarrow b \bar{b} \phi\left(\rightarrow \tau^{+} \tau^{-}\right)+X\right] \propto \hat{h}_{b}^{2} \times \hat{B}_{\tau},
$$

where $\hat{h}_{b}=h_{b}^{C P}, \overline{h_{b}}$, or $\widetilde{h_{b}}$ depending on $\phi$, and $\hat{B}_{b}$ and $\hat{B}_{\tau}$ are the corresponding branching ratios of the $\phi$ decay into bottom quarks and $\tau$ leptons, which are computed using the modified couplings $\hat{h}_{b}$ and $\hat{h}_{\tau}$. In general, while the four $b$ final state, Eq. (4.1), is strongly affected by $\Delta\left(m_{b}\right)$, the $b \bar{b} \tau^{+} \tau^{-}$final state [25], Eq. (4.2), is only mildly affected due to a cancellation of the dependence of the production cross section times branching ratios on this factor.

\section{Comments on the CP-even Higgs boson masses at large values of $\tan \beta$}

One interesting feature of the large $\tan \beta$ regime is that the $C P$-odd and one of the two $C P$-even Higgs bosons have similar masses and couplings. One might be tempted to take the signal from $b \bar{b} A$ production and decay and double it to account for the other non-SM-like Higgs boson. However, this approximation is optimistic, and not necessary. For ex- 
ample, when both $|\bar{\mu}|$ and $|\tilde{a}| \gtrsim \mathcal{O}(1)$, this might be a poor approximation [26]. Indeed, the $C P$-even Higgs boson with similar properties to the $C P$-odd one has a mass approximately equal to $\mathcal{M}_{11}$, with the form

$$
\mathcal{M}_{11}^{2} \simeq m_{A}^{2}-\frac{m_{t}^{4}}{16 \pi^{2} \mathrm{v}^{2}} \bar{\mu}^{2} \tilde{a}^{2}
$$

where we have omitted the two-loop corrections, Eq. (2.3). A particularly interesting case to analyze is the maximal mixing case, $\tilde{a}^{2}=6$, when the radiative corrections to $m_{\phi^{\mathrm{SM}}}^{2}$ are maximized and $\mathcal{M}_{12}^{2}$ receives only small radiative corrections for moderate or large values of $m_{A}$. For small mass differences compared to the average mass, one gets approximately

$$
m_{A}-m_{h} \simeq \frac{3 m_{t}^{4}}{8 \pi^{2} \mathrm{v}^{2}} \frac{\bar{\mu}^{2}}{2 m_{A}} \quad\left(\tilde{a}^{2}=6\right)
$$

For $|\bar{\mu}| \geqslant 1$, both $C P$-even Higgs boson masses can be significantly different from the $C P$-odd one. Figure 12 shows the minimal and maximal mass difference of the $C P$-even Higgs boson mass $m_{h}\left(m_{H}\right)$ with the $C P$-odd Higgs boson mass, for values of $\sin ^{2}(\beta-\alpha)<0.5\left[\sin ^{2}(\beta-\alpha)>0.5\right]$, in the maximal mixing case and $|\bar{\mu}|=1$. A scan was performed over values of $m_{A}>80 \mathrm{GeV}$. As is clear from the above expression, the maximal and minimal mass differences are obtained for the minimal and maximal values of $m_{A}$ chosen. For instance, for values of $m_{A} \simeq 80 \mathrm{GeV}, \tilde{a}=\sqrt{6}$ and $\bar{\mu}$ $= \pm 1$, one obtains a mass difference of about $5 \mathrm{GeV}$ for large $\tan \beta$, which coincides with the results presented in the figure. Had we scanned over lower values of $m_{A}$, the mass difference would have increased. In our analysis we consider the separate signals from $A$ and the $C P$-even-like Higgs boson with similar masses and couplings as the $C P$-odd Higgs boson.

\section{Numerical results for the $b \bar{b} \phi$ channel}

Several studies have examined this channel. ${ }^{10}$ For our numerical results, we rely on the analysis performed by the Higgs Working Group of the Physics at Run IISupersymmetry/Higgs Workshop [19] for the $b \bar{b} H^{\mathrm{SM}}$ channel. The results for this channel are preliminary, but are in general agreement with previous studies. The exact numbers are based on a combination of the CDF and D0 data, with no systematic errors, and using the CDF Run I mass resolution. The estimate of the number of signal events is sensitive to unknown higher-order QCD corrections, and the overall rate might be off by a factor of 2 , based on our experience with

\footnotetext{
${ }^{10}$ This process was first considered at the Tevatron based on a three jet analysis [27]. Later, it was reconsidered based on a four jet analysis [28]. We corrected two critical errors in that analysis, and the modified results of Ref. [28] presented in Ref. [29] are now in general agreement with our results when comparable.
}

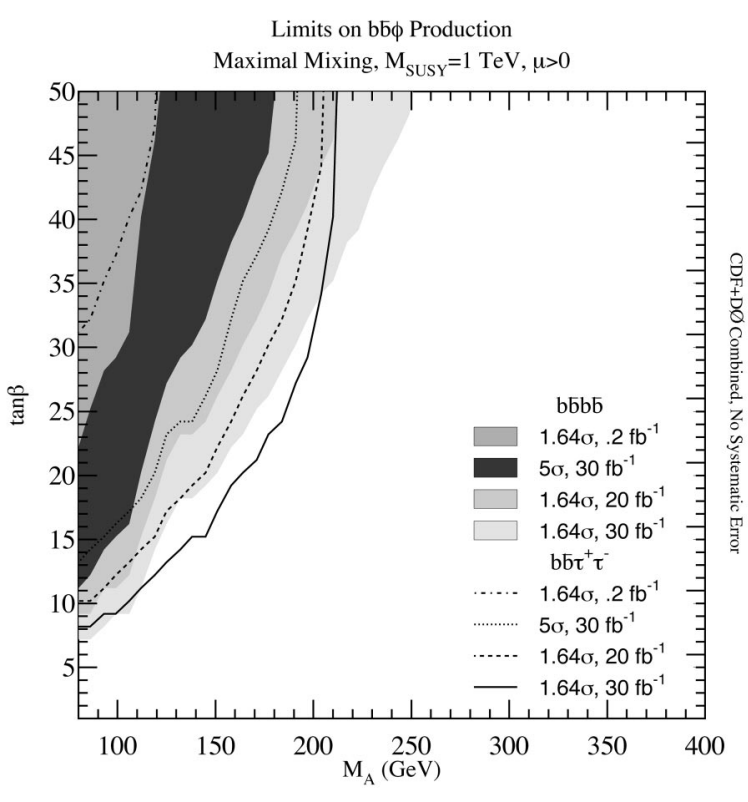

FIG. 13. 95\% C.L. exclusion and $5 \sigma$ discovery contours for $b \bar{b} \phi$ production at the Tevatron in the MSSM for maximal mixing and $\mu>0$. The shaded areas correspond to different exclusion and discovery contours at different integrated luminosities for the $b \bar{b} b \bar{b}$ final state. The different lines show the same for the $b \bar{b} \tau^{+} \tau^{-}$final state. The results assume a combination of the CDF and $\mathrm{D} \emptyset$ data, with no systematic errors, and using the CDF run I mass resolution. The exact contours are sensitive to unknown higher-order QCD corrections, as explained in the text.

heavy quark pair production. The background is estimated from run I data, but scaled by a factor of 1.3 to account for the increased beam energy in run II.

The $95 \%$ C.L. exclusion and $5 \sigma$ discovery potentials of the $b \bar{b} b \bar{b}$ channel are illustrated in Fig. 13 for different total integrated luminosities and for the case of maximal mixing and $\mu>0$. These results imply that a $C P$-odd Higgs boson (and its partner with similar properties) can be discovered with $30 \mathrm{fb}^{-1}$ of data at large $\tan \beta$ if $m_{A} \lesssim 180 \mathrm{GeV}$. For the same integrated luminosity, an exclusion contour can cover from $m_{A} \simeq 80 \mathrm{GeV}$ and $\tan \beta \simeq 7$ up to $m_{A}$ $\simeq 250 \mathrm{GeV}$ and large $\tan \beta \simeq 50$. However, these conclusions assume vanishing SUSY corrections to the bottom mass $\left[\Delta\left(m_{b}\right)=0\right]$. Figure 14 shows the sensitivity of these results to SUSY corrections at large $\tan \beta$. The lines in Fig. 14 show the variation of the $5 \sigma$ discovery contour with $30 \mathrm{fb}^{-1}$ for $\Delta m_{b}=K \times \tan \beta$, with $K= \pm 0.005$ and \pm 0.01 . There is a similar variation in the exclusion contours. Clearly, it is difficult to make a definitive statement about the reach of the Tevatron in the $b \bar{b} b \bar{b}$ final state with limited knowledge of the size of the bottom mass corrections $\Delta\left(m_{b}\right)$ which depend on the sparticle masses and mixings. It is important to realize that, for negative values of $K$ and large values of $\tan \beta$, the cross section increases due to a large increase in the bottom Yukawa coupling $h_{b}$, but this Yukawa coupling may become too large to make a perturbative analysis possible. This would happen, for instance for values of $|K| \geq 0.015$ and $\tan \beta \gtrsim 50$. 


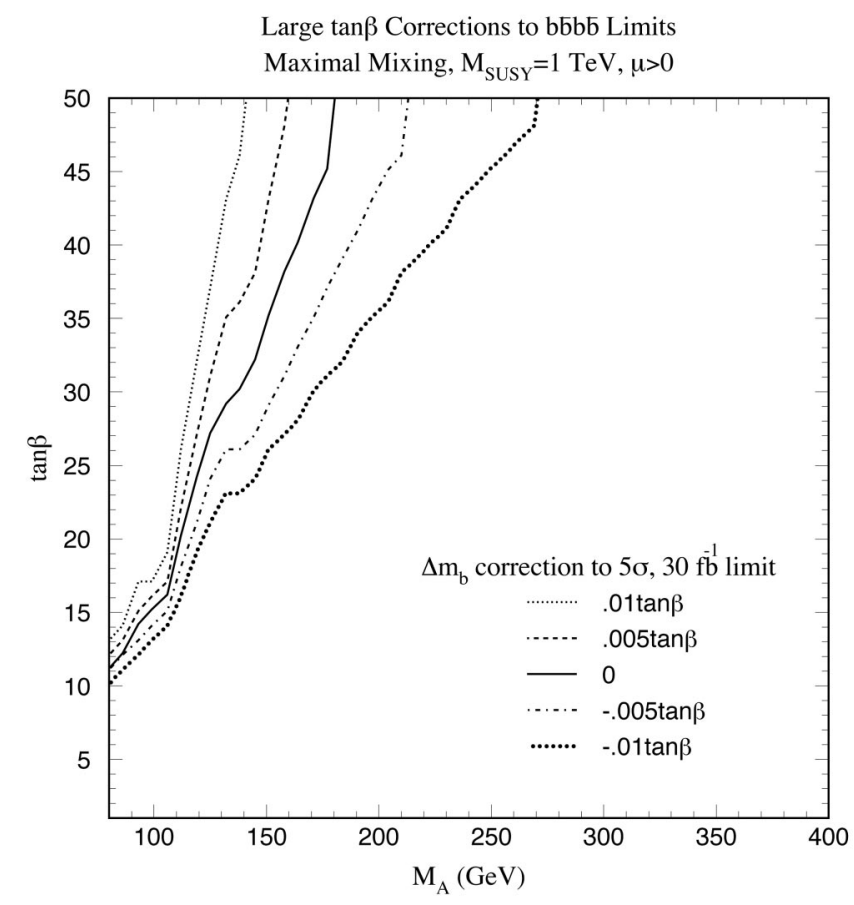

FIG. 14. The effect of radiative corrections $\Delta\left(m_{b}\right)=(0$, $\pm 0.005, \pm 0.01) \tan \beta$ to the $5 \sigma$ discovery contour of Fig. 13 .

One of the generic conclusions of this study is that, in the large $\tan \beta$ regime, this process may be useful to test regions of parameter space which will remain otherwise uncovered. In general, however, the reach in the $m_{A}$-tan $\beta$ plane is reduced to a relatively small region for values of $m_{A}$ of the order of the weak scale. The exact discovery and exclusion potentials depend strongly on the finite SUSY corrections to the bottom mass, which can be very large.

\section{Simulation of $b \bar{b} \phi(\rightarrow \tau \tau)$ signal and backgrounds and results}

We have also considered the possibility of detecting the Higgs boson decays into $\tau^{+} \tau^{-}{ }^{11}$ Since $\Delta\left(m_{\tau}\right)$ is expected to be small, the $b \bar{b} \tau^{+} \tau^{-}$channel is not likely to be very sensitive to SUSY-induced, large $\tan \beta$ corrections. This follows from Eq. (4.2) in the limit that the total width of the Higgs boson is dominated by the $b \bar{b}$ partial width. We present a preliminary study here, but more work needs to be done to understand the feasibility of this channel. With our present understanding, Fig. 13 shows that the reach of this channel is comparable to or even slightly better than the $b \bar{b} b \bar{b}$ channel, and one may expect much room for improvement.

To study this process at the Tevatron, we considered only the $\tau_{1}(\rightarrow l+X)$ and $\tau_{2}(\rightarrow j+X)$ decays of the $\tau_{1} \tau_{2}$ pair, where $l=e$ or $\mu$. This combination yields a triggerable lepton, a narrow jet, and two $b$ quarks in the final state. It also

\footnotetext{
${ }^{11}$ This channel was previously considered for Run I [25]. However, due to a numerical error, the reach in $\tan \beta$ was largely overestimated in that work [32].
}

has the largest branching ratio. The physics backgrounds are assumed to be $b \bar{b} Z(\rightarrow \tau \tau)$ and $t \bar{t}$ production. The signal $b \bar{b} \phi(\rightarrow \tau \tau)$ and the background $b \bar{b} Z(\rightarrow \tau \tau)$ cross sections are estimated from a parton-level simulation based on MADGRAPH [30] matrix elements. All matrix elements are evaluated at leading-order, using leading-order $\alpha_{s}$, leadingorder parton distribution functions (CTEQ3L), and a common scale $\sqrt{\hat{s}} / 2$, where $\sqrt{\hat{s}}$ is the partonic center-of-mass energy. ${ }^{12}$ The Higgs boson and $Z$ boson resonances are treated in the narrow width approximation. Next-to-leading order QCD corrections to these processes are expected to be important, but they have not yet been calculated. Since the $b \bar{b}$ production cross section at the Tevatron at NLO is almost doubled from the LO result, as a first estimate the signal and backgrounds in this study are multiplied by a factor of 2 [31]. This assumption will need to be considered in more detail elsewhere. When Gaussian statistics apply, this increases the significance $(S / \sqrt{B})$ of a signal by $\sqrt{2}$. In addition, the $\tau$ polarization information was included for all $\tau$ decays through the TAUOLA Monte Carlo program [33]. The $t \bar{t}$ background was simulated using PYTHIA 6.1 [34] with the default settings, and forcing $W$ decays into $e, \mu$, or $\tau$. For this analysis, the $b$ parton is treated as a $b$ jet, but the $\tau$ jet is constructed from final state particles ( $\pi, K$, etc.). The $\boldsymbol{E}_{T}$ is constructed from the real neutrinos from $W$ boson and $\tau$ decays.

Because the number of backgrounds is smaller than for the $b \bar{b} b \bar{b}$ channel and the signal and $Z$ background have similar characteristics, it is assumed that the acceptance cuts can be looser. The basic cuts are $2 b$ partons with $p_{T}^{b}$ $>10 \mathrm{GeV},\left|\eta^{b}\right|<2,1 e$ or $\mu$ with $p_{T}^{l}>10 \mathrm{GeV},\left|\eta^{l}\right|<2$, $1 \tau$-jet with $p_{T}^{j}>15 \mathrm{GeV},\left|\eta^{j}\right|<2$, and $R_{i j}>0.6$, where $i, j$ sum over $b$ 's, $l$, and $\tau$ jet. After these cuts, the $t \bar{t}$ events produce the largest background. However, the jets and leptons from $t$-quark decays are much harder and produce much more $\mathbb{E}_{T}$ than the typical signal event. The further cuts $p_{T}^{b}$ $<60 \mathrm{GeV}$ and $\mathbb{E}_{T}<80 \mathrm{GeV}$ are imposed to reduce this background. For the final numbers, the CDF $\tau$-jet reconstruction efficiency ranging from approximately 0.3 to 0.6 is used [35], as well as a double $b$-tag efficiency ${ }^{13}$ of 0.45 and a triggering efficiency of unity.

The signal is defined by a simple counting experiment, without reference to a mass window. Several possible improvements could greatly increase the potential of the $b \bar{b} \tau \tau$ signal. First, with adequate $\boldsymbol{E}_{T}$ resolution, a mass peak can be partially reconstructed. This could distinguish the signal from the background for $m_{\phi} \gg M_{Z}$. Second, the second largest branching ratio for the decay of a $\tau$ pair is when both $\tau$ 's decay to jets. While this channel would greatly enhance the signal, it requires a detailed background and triggering analysis beyond the scope of this work.

\footnotetext{
${ }^{12}$ The scale dependence of these results is estimated to be $25 \%$ [29].

${ }^{13}$ This estimate is based on the double tag efficiency of Ref. [5].
} 


\section{CONSTRAINTS FROM THE DECAY $B \rightarrow s \gamma$}

As shown above, the couplings of the $C P$-even Higgs bosons to bottom quarks depend strongly on $\Delta\left(m_{b}\right)$. In the MSSM framework, positive or negative corrections are possible. However, in some specific models, the sign of the correction is correlated with the supersymmetric contribution to the amplitude of the decay process $B(b \rightarrow s \gamma)$, which, at large values of $\tan \beta$, is proportional to $A_{t} \times \mu \tan \beta$ [36]. This is the case, for instance, in the minimal supergravity model, with unification of the three gaugino masses. For moderate and large values of $\tan \beta$ [37,22],

$$
A_{t} \simeq \frac{A_{0}}{4}-1.5 M_{1 / 2},
$$

where $A_{0}$ and $M_{1 / 2}$ are the boundary conditions for $A_{t}$ and the gaugino masses, respectively, at the grand unified theory (GUT) scale. Unless $A_{0} \approx 6 M_{1 / 2}$, we have $A_{t} \times M_{\tilde{g}}<0$. Moreover, it has been shown [22] that unless $A_{0}$ and $m_{0}$ are much larger than $M_{1 / 2}$, the expression for $\Delta\left(m_{b}\right)$ in Eq. (3.4) is dominated by the gluino contributions, which are proportional to $\mu \times M_{\tilde{g}}$. The top-squark-induced corrections, proportional to the trilinear parameter $A_{t}$, are smaller than the gluino-induced ones and tend to reduce the total bottom mass corrections. Hence, the sign of the bottom mass corrections is determined by the gluino-sbottom loop contribution, which is opposite in sign to the chargino-top-squark corrections to the $b \rightarrow s \gamma$ decay rate. Cancellation of the positive contribution of the charged Higgs boson $H^{+}$to $B(b \rightarrow s \gamma)$ requires $A_{t} \times \mu<0$, so that in these models the bottom mass corrections $\Delta\left(m_{b}\right)>0$. Positive corrections to the bottom mass $\left[\Delta\left(m_{b}\right)>0\right]$ reduce the effective bottom Yukawa coupling with respect to the tree level value, Eq. (1.1), which reduces the discovery and exclusion potential of a Higgs boson in the $b \bar{b} b \bar{b}$ final state at the Tevatron collider (see Fig. 14). As discussed above, positive mass corrections have also a negative effect on the exclusion and discovery potential of a $C P$-even Higgs boson in the $V \phi$ channel (see Figs. 10 and $11)$.

In general, in the absence of flavor violating couplings of the down squarks to gluinos, the $B(b \rightarrow s \gamma)$ constraint on the possible values of $m_{A}$ and the top squark mass parameters becomes strong for large values of $\tan \beta$. For low values of the $C P$-odd Higgs boson mass, positive values of $\mu \times A_{t}$ are disfavored by the data. Even for negative values of $\mu \times A_{t}$, when $m_{A} \simeq M_{Z}$, the suppression induced by the chargino-top squark contributions tends to be too small to cancel the large charged Higgs boson enhancement, unless $\left|\bar{A}_{t} \times \bar{\mu}\right| \times \tan \beta$ becomes large. This cannot be achieved by pushing the value of the $\mu$ parameter to values larger than $M_{S}$, since this would increase the chargino masses and lower the loop effect. Hence, large values of $\tan \beta$ and $\left|A_{t}\right|$ are preferred.

The above mentioned constraints on the top squark mass parameters for low values of $m_{A}$ can be avoided in the presence of a nontrivial down squark flavor mixing. In particular, non-negligible mixing parameters between the second and third generation of down squarks can contribute to the $b$ $\rightarrow s \gamma$ decay rate via gluino-squark loop induced processes.
For instance, if the gluino contributions were the only ones leading to the $b \rightarrow s \gamma$ decay rate, the branching ratio would be given by [38]

$$
B(b \rightarrow s \gamma) \simeq \frac{2 \alpha_{3}^{2} \alpha_{\mathrm{em}}}{81 \pi^{2} M_{S}^{4}} m_{b}^{3} \tau_{B} M_{\tilde{g}}^{2} F^{2}(x)\left|\frac{\Delta_{23}^{d}}{M_{S}^{2}}\right|^{2},
$$

where $\Delta_{23}$ is the value of the off-diagonal sbottom-sstrange left-right term in the down squark squared mass matrix (which we assume to be equal to the right-left one), $\tau_{B}$ $\simeq 1.5 \times 10^{-12} s, x=M_{\tilde{g}} / M_{S}$, and

$$
F(x)=4\left(\frac{1+4 x-5 x^{2}+4 x \ln (x)+2 x^{2} \ln (x)}{8(1-x)^{4}}\right) .
$$

This expression ignores the potentially relevant contributions coming from a left-left down squark mixing $[38,40]$. Observe that, for $M_{\tilde{g}}=\mathcal{O}\left(M_{S}\right)$ and $M_{S}=\mathcal{O}(1 \mathrm{TeV})$, the contribution of the gluino mediated diagram to the $b \rightarrow s \gamma$ branching ratio is of the order of $\left(\Delta_{23}^{d} / M_{S}^{2}\right)^{2}$. Hence, even a small left-right mixing $\Delta_{23}^{d}$ of order of $10^{-2} \times M_{S}^{2}$ can induce important corrections to the amplitude of this decay rate. It is straightforward to show that these low values of $\Delta_{23}$ do not have an immediate impact on the Higgs boson sector.

In the presence of nontrivial flavor mixing in the down squark sector, large corrections to the amplitude of the $b$ $\rightarrow s \gamma$ decay rate may be induced. These corrections may be helpful in determining values of $B(b \rightarrow s \gamma)$ consistent with experimental data for small values of $m_{A}$ and/or positive values of $\mu \times A_{t}$. For the above reasons, in our presentation, we have decided to keep the results for both signs of $\bar{A}_{t}$ $\times \bar{\mu}$. The reader must keep in mind, however, that positive values of this parameter for low values of $m_{A}$ would imply a more complicated flavor structure that the one appearing in minimal gauge mediation or supergravity schemes. Observe that the contributions of the charged Higgs boson and topsquark loops to $b \rightarrow s \gamma$, should be computed including the effect of the bottom mass corrections $\Delta\left(m_{b}\right)$ in the definition of the bottom Yukawa coupling $h_{b}$. A next-to-leading-order SUSY QCD calculation of $\mathrm{B}(b \rightarrow s \gamma)$ can be found in Ref. [39].

\section{CONCLUSIONS}

We have presented a study of some of the MSSM Higgs boson signatures of relevance to the Tevatron collider. We first analyzed the possibility of finding the lightest or heaviest $C P$-even Higgs bosons in the $V \phi$ channel, where $V$ $=W$ or Z. Quite generally, for moderate and large $\tan \beta$, either the lightest or the heaviest $C P$-even Higgs boson has SM-like couplings to the vector bosons. Therefore, most of the $\tan \beta-m_{A}$ plane is covered in this channel by producing the corresponding Higgs boson with mass below $130 \mathrm{GeV}$, provided there is sufficient integrated luminosity, of the order of $30 \mathrm{fb}^{-1}$. However, for $m_{A} \approx m_{\phi^{\mathrm{SM}}} \simeq 110-130 \mathrm{GeV}$ and large $\tan \beta$, neither $h$ nor $H$ has SM-like couplings to the vector gauge bosons and the coverage is decreased. This problem is more pronounced for large values of the top 
squark masses and mixing parameters. For smaller values of $\tan \beta \simeq 5$, this problematic region extends to smaller values of $m_{A}$. In these cases, very large luminosity, above $30 \mathrm{fb}^{-1}$, will be needed or the contribution of other production processes will be necessary to assure full coverage. To cover the region of $\tan \beta>10$ and $m_{A} \approx m_{\phi^{\mathrm{SM}}}$, we have combined the two $C P$-even Higgs boson signals when their masses are close to each other. Another possibility may be to explore the $h A$ and $H A$ channels, which will suffer from similar suppression factors in the production cross sections $\left[\sin ^{2}(\beta\right.$ $\left.-\alpha) \simeq \cos ^{2}(\beta-\alpha) \simeq \mathcal{O}(0.5)\right]$, but may be combined with the $V \phi$ production process.

Furthermore, the branching ratio for the decay of the $C P$-even Higgs bosons into bottom quarks can be very different from the standard model one. In particular, this takes place for moderate and large $\tan \beta$, when the off-diagonal elements of the Higgs boson mass matrix can be strongly modified by radiative corrections induced mainly by top squark loops. We derived an approximate expression in terms of the MSSM parameters to clarify when this occurs, and provided examples when the decays into bottom quarks are suppressed in a large region of parameter space, thereby negatively affecting the Tevatron reach.

We have also emphasized that, due to supersymmetry breaking effects, the values of the bottom and $\tau$ Yukawa couplings to the $C P$-even Higgs bosons may be different from the ones computed including only standard QCD corrections. Indeed, nondecoupling effects induced by supersymmetry breaking can become particularly important for large $\tan \beta$, leading to modifications in the Higgs boson discovery and exclusion potentials at the Tevatron. For instance, if the bottom mass corrections $\Delta\left(m_{b}\right)$ are of order 1 , the decay of the standard model-like Higgs boson into $\tau^{+} \tau^{-}$ may be enhanced, while decays to $b \bar{b}$ are suppressed. These supersymmetry breaking effects on the bottom and the $\tau$ Yukawa couplings can also have an impact in the phenomenology of the charged Higgs boson. In particular, they can be relevant for determining the Tevatron limits on top decays into charged Higgs bosons at large $\tan \beta[24,41]$.

The bottom Yukawa coupling corrections are particularly important for the $b \bar{b} \phi$ process, because the production cross section is proportional to the square of the bottom Yukawa coupling. We performed a phenomenological study to investigate the relevance of these corrections. Even with large luminosity factors, of the order of $30 \mathrm{fb}^{-1}$, and negative bottom mass corrections, which enhance the production rate, the Tevatron can discover a $C P$-odd Higgs boson (together with a $C P$-even Higgs boson with mass and couplings similar to it) only if its mass is not larger than about 200-300 $\mathrm{GeV}$. The reach is only efficient for moderate or large values of $\tan \beta$. Such values for the $C P$-odd Higgs boson mass give positive contributions to $B(b \rightarrow s \gamma)$, and the discovery of such a Higgs boson would constrain the masses and mixing angles of the top squarks unless a nontrivial mixing between the second and third generation down squarks is present.

The computation of the Higgs boson mass matrix elements considered in this article [8] is still affected by theo- retical uncertainties, most notably, those associated with the two-loop, finite, threshold corrections to the effective quartic couplings of the Higgs potential. Recently, a partial, diagrammatic, two-loop computation of the Higgs boson mass has been performed [42]. In the limit of large $m_{A}$, these additional contributions lead to a slight modification of the dependence of the lightest $C P$-even Higgs boson mass on the top squark mixing parameters. For instance, although the upper bound on the lightest $C P$-even Higgs boson mass for squark masses of approximately $1 \mathrm{TeV}$ is approximately the same as the one obtained to next-to-leading-order accuracy (as done in this work), the upper bound on the Higgs boson mass is reached for values of $|\tilde{a}| \simeq 2$ instead of $|\tilde{a}|=\sqrt{6}$, and has a weak dependence on the sign of $\tilde{a}$. A diagrammatic computation of the two-loop corrections induced by the top Yukawa coupling, which are included at the leadinglogarithmic level in our computation is, however, still lacking [43].

Summarizing, at present, the $V \phi$ channel with the $V$ decaying leptonically and the Higgs boson decaying into $b$ quarks remains the golden mode to test the MSSM Higgs sector at the Tevatron. The other channel we have analyzed, $b \bar{b} \phi$ production with the subsequent decay of $\phi$ into $b$ quarks and $\tau$ leptons, proves to be very useful to cover regions of large $\tan \beta$ and small to moderate $m_{A}$ up to about $250 \mathrm{GeV}$. However, the reach in these channels requires a large total integrated luminosity. Because of this, other production processes and Higgs boson decay modes need to be carefully investigated if we want to fully probe the MSSM Higgs boson sector at the Tevatron. We have identified regions of parameter space where the Higgs boson decay into $b \bar{b}$ is strongly suppressed. In these regions, other search techniques will have to be considered, due to the presence of enhanced decays to $W^{*} W^{*}, g g$, and $c \bar{c}$ final states. Clearly, there is a motivation to study these final states, and in particular the $W^{*} W^{*}$ one, even for lighter Higgs bosons for which the SM Higgs boson decay rate is strongly suppressed. In addition other production processes such as the associated production of $h A$ or $H A$ with the subsequent decays into $b$ quarks and $\tau$ leptons may also be useful.

A careful study of all different possibilities, which may be relevant in different regions of parameter space, and the combination of channels may allow a full coverage of the MSSM parameter space with luminosities achievable at the Tevatron. If that is the case, the Tevatron can discover a light Higgs boson which might be beyond the presently expected LEP2 reach for generic values of the supersymmetric mass parameters. Most importantly, the detection of one or more Higgs bosons at the Tevatron will give very valuable information about the Higgs and top squark sectors of the MSSM.

In the final stages of this work, two preprints appeared on related topics. One addressed the issue of the $W \phi$ reach of the Tevatron collider [44]. The other commented on the possible effects of large $\tan \beta$ corrections to $b \bar{b} \phi$ production at hadron colliders [29]. In the special cases when the analyses are comparable we tend to agree with their results, although the authors of Ref. [44] do not see any visible dependence on 
the sign of $\mu \times \tilde{a}$. The present work goes beyond those studies by providing a detailed numerical and theoretical analysis of the dependence of the Tevatron discovery potential on the MSSM parameter space.

\section{ACKNOWLEDGMENTS}

C.E.W. thanks the hospitality of the theory groups at Fermilab and at the University of Buenos Aires, where part of this work was completed. M.C. and C.E.W. are grateful to the Rutherford Laboratory, as is S.M. to the Aspen Center for Physics. We also acknowledge discussions with K. Matchev and T. Tait. The research of M.C. is supported by the Fermi National Accelerator Laboratory, which is operated by the Universities Research Association, Inc., under Contract No. DE-AC02-76CHO300. The work of S.M. is supported in part by the U.S. Dept. of Energy, High Energy Physics Division, under Contract No. W-31-109-ENG-38.
[1] M. Carena, P. Zerwas, and the Higgs Physics Working Group, ' 'Physics at LEP2,', edited by G. Altarelli, T. Sjöstrand, and F. Zwirner, CERN Report No. 96-01, Vol. 1.

[2] A. Strange, W. Marciano, and S. Willenbrock, Phys. Rev. D 49, 1354 (1994); 50, 4491 (1994).

[3] S. Mrenna and G. L. Kane, Report No. CALT-68-1938, hep-ph/9406337.

[4] 'Future ElectroWeak Physics at the Fermilab Tevatron,', Report No. Fermilab-Pub-96/082, edited by D. Amidei and R. Brock, 1996.

[5] S. Kim, S. Kuhlmann, and W.-M. Yao, in "Proceedings of the 1996 DPF/DPB Summer Study on New Directions for High Energy Physics,"' 1996 (unpublished).

[6] W. M. Yao, in "Proceedings of the 1996 DPF/DPB Summer Study on New Directions for High Energy Physics," 1996 (unpublished).

[7] D. Reid, talk at the XXXIII Rencontres de Moriond (Electroweak Interactions and Unified Theories), Les Arcs, France, 1998; LEP Electroweak Working Group, Report No. LEPEWWG/97-01.

[8] M. Carena, J.-R. Espinosa, M. Quiros, and C. E. M. Wagner, Phys. Lett. B 355, 209 (1995); M. Carena, M. Quiros, and C. E. M. Wagner, Nucl. Phys. B461, 407 (1996).

[9] H. Haber, R. Hempfling, and A. H. Hoang, Z. Phys. C 57, 539 (1997)

[10] A. Djouadi, J. Kalinowski, and M. Spira, Comput. Phys. Commun. 108, 56 (1998).

[11] R. Hempfling and A. Hoang, Phys. Lett. B 331, 99 (1994); J. Kodaira, Y. Yasui, and K. Sasaki, Phys. Rev. D 50, 7035 (1994).

[12] J. Casas, J. R. Espinosa, M. Quiros, and A. Riotto, Nucl. Phys. B436, 3 (1995).

[13] J. Ellis, T. Falk, K. Olive, and M. Schmitt, Phys. Lett. B 388, 97 (1996); 413, 355 (1997); S. A. Abel and B. C. Allanach, ibid. 431, 339 (1998); J. A. Casas, J.-R. Espinosa, and H. E. Haber, Nucl. Phys. B526, 3 (1998).

[14] M. Carena, P. Chankowski, S. Pokorski, and C. E. M. Wagner, Phys. Lett. B 441, 205 (1998).

[15] M. Baer and J. Wells, Phys. Rev. D 57, 4446 (1998); W. Loinzaz and J. D. Wells, Phys. Lett. B 445, 178 (1998).

[16] S. Mrenna, Perspectives on Higgs Physics II, edited by G. L. Kane (World Scientific, Singapore, 1997), p. 131.

[17] R. K. Ellis and S. Veseli, Phys. Rev. D 60, 011501 (1999).

[18] A. Belyaev, E. Boos, and L. Dudko, Mod. Phys. Lett. A 10, 25 (1995).
[19] " Report of the Physics at Run II Supersymmetry/Higgs Workshop," Fermilab, 1999, edited by M. Carena and J. Lykken (in preparation).

[20] T. Han and R. J. Zhang, Phys. Rev. Lett. 82, 25 (1999).

[21] L. Hall, R. Rattazzi, and U. Sarid, Phys. Rev. D 50, 7048 (1994); R. Hempfling, ibid. 49, 6168 (1994).

[22] M. Carena, M. Olechowski, S. Pokorski, and C. E. M. Wagner, Nucl. Phys. B426, 269 (1994).

[23] D. Pierce, J. Bagger, K. Matchev, and R. Zhang, Nucl. Phys. B491, 3 (1997).

[24] J. A. Coarasa, R. A. Jimenez, and J. Sola, Phys. Lett. B 389, 312 (1996); R. A. Jimenez and J. Sola, ibid. 389, 53 (1996).

[25] M. Drees, M. Guchait, and P. Roy, Phys. Rev. Lett. 80, 2047 (1998).

[26] R. Hempfling, Phys. Lett. B 296, 121 (1992); J. Rosiek and A. Sopczak, ibid. 341, 419 (1995).

[27] J. Dai, J. F. Gunion, and R. Vega, Phys. Lett. B 387, 801 (1996).

[28] J. L. Diaz-Cruz, H.-J. He, T. Tait, and C.-P. Yuan, Phys. Rev. Lett. 80, 4641 (1998).

[29] C. Balazs, J. L. Diaz-Cruz, H. J. He, T. Tait, and C.-P. Yuan, Phys. Rev. D 59, 055016 (1999).

[30] W. F. Long and T. Stelzer, Comput. Phys. Commun. 81, 357 (1994).

[31] M. Mangano, P. Nason, and G. Ridolfi, Nucl. Phys. B373, 295 (1992).

[32] M. Drees (private communication).

[33] S. Jadach, Z. Was, R. Decker, and J. H. Kuhn, Comput. Phys. Commun. 76, 361 (1993).

[34] T. Sjöstrand, Comput. Phys. Commun. 82, 74 (1994).

[35] CDF Collaboration, F. Abe et al., Phys. Rev. Lett. 78, 2906 (1997).

[36] S. Bertolini, F. Borzumati, A. Masiero, and G. Ridolfi, Nucl. Phys. B353, 591 (1991); R. Barbieri and G. Giudice, Phys. Lett. B 309, 86 (1993).

[37] See, for example, C. Kounnas, I. Pavel, G. Ridolfi, and F. Zwirner, Phys. Lett. B 354, 322 (1995).

[38] F. Gabbiani, E. Gabrielli, A. Masiero, and L. Silvestrini, Nucl. Phys. B477, 321 (1996).

[39] M. Ciuchini, G. Degrassi, P. Gambino, and G. F. Giudice, Nucl. Phys. B527, 21 (1998); B534, 3 (1998); F. Borzumati and C. Greub, Phys. Rev. D 58, 074004 (1998).

[40] See, for example, T. Blazek and S. Raby, Phys. Rev. D 59, 095002 (1999). 
[41] J. A. Coarasa, J. Guasch, J. Sola, and Hollik, Phys. Lett. B 442, 326 (1998).

[42] S. Heinemeyer, W. Hollik, and G. Weiglein, Phys. Rev. D 58, 091701 (1998); Phys. Lett. B 440, 96 (1998).
[43] For a very recent computation using two loop effective potential methods, see R.-J. Zhang, Phys. Lett. B 447, 89 (1999).

[44] H. Baer, B. W. Harris, and X. Tata, Phys. Rev. D 59, 015003 (1998). 